Research Article

\title{
Application of Some Fixed-Point Theorems in Orthogonal Extended S-Metric Spaces
}

\author{
Mohammad Mahdi Rezaei, ${ }^{1}$ Shaban Sedghi $\left(\mathbb{D},{ }^{1}\right.$ and Vahid Parvaneh ${ }^{2}{ }^{2}$ \\ ${ }^{1}$ Department of Mathematics, Qaemshahr Branch, Islamic Azad University, Qaemshahr, Iran \\ ${ }^{2}$ Department of Mathematics, Gilan-E-Gharb Branch, Islamic Azad University, Gilan-E-Gharb, Iran \\ Correspondence should be addressed to Shaban Sedghi; sedghi.gh@qaemiau.ac.ir
}

Received 29 April 2021; Accepted 7 October 2021; Published 29 October 2021

Academic Editor: Xiaolong Qin

Copyright (C) 2021 Mohammad Mahdi Rezaei et al. This is an open access article distributed under the Creative Commons Attribution License, which permits unrestricted use, distribution, and reproduction in any medium, provided the original work is properly cited.

In this study, we obtain some coincidence point theorems for weakly $O$ - $\alpha$-admissible contractive mappings in an orthogonal extended $S$-metric space. An example and an application are provided to illustrate the usability of the obtained results. Our results generalize the results of several studies from metric and $S$-metric frameworks to the setting of orthogonal extended $S$-metric spaces.

\section{Introduction}

In 1922, Banach [1] proposed a theorem known as the Banach fixed point theorem (or Banach contraction principle or BCP for short) to prove the existence of solutions for nonlinear operator equations and integral equations. Since then, it has become a very popular tool due to its simplicity and practicality in solving a variety of problems such as control theory, economic theory, nonlinear analysis, and global analysis. Later, there is a large body of literature on the applications, generalizations, and expansions of this theorem. In [2], for example, the authors introduced the definition of a cyclic p-contraction pair for single-valued mappings and presented some best proximity point results for such mappings on a proximally complete pair of subsets in a metric space. In [3], Dehici and Redjel obtained some fixed-point results for nonexpansive mappings defined on an intersection of a finite number of closed bounded and convex nonempty subsets in Banach spaces. These research studies have been done by several authors in different directions, for example, by weakening the hypothesis, using different settings, considering different contractions, and so on.

Many mathematical problems require finding distances between two or more objects that are not easy to measure exactly in general. There are different approaches to get the right concept of a metric structure. Due to the need to create an appropriate framework for modeling several salient problems of a practical nature, the study of metric spaces has attracted the attention of many researchers. In the last few decades, a number of generalizations of metric spaces in several articles, such as 2-metric spaces [4], G-metric spaces [5], $D^{*}$-metric spaces [4], partial metric spaces [6], and cone metric spaces [7], have been illustrated.

Sedghi et al. [8] introduced the concept of an $S$ metric space and proved that this concept is a generalization of $G$-metric space and $D^{*}$-metric space. Also, they have proven some properties on $S$-metric spaces and some fixed-point theorems for mappings defined on an $S$-metric space.

The concept of $b$-metric space, as one of the useful generalizations of standard metric spaces, was first used by Bakhtin in [9] and Czerwik in [10]. This idea has been extended by Parvaneh and Ghoncheh [11] to the $p$-metric settings.

The concept of weak contraction mapping was introduced by Alber and Guerre-Delabrere in [12] in the arrangement of Hilbert spaces. Rhoades [13] proved that every weak contraction mapping on a complete metric space has a unique fixed point. Zhang and Sang [14] have generalized this concept to weakly contractive pairs. 
In this study, we prove some coincidence point results for $\alpha$-admissible $(\pi, \Phi)$-contractive mappings in orthogonal extended $S$-metric spaces where we were motivated by the work in [15]. Using an auxiliary function, commonly referred to as $\alpha$, in the article [16] by Samet et al., which continued in several articles, we want to define some related concepts such as orthogonal $\alpha$-compatibility and orthogonal $\alpha$-regularity.

\section{Preliminaries}

From now on,

$$
\Omega=\left\{\omega:[0, \infty) \longrightarrow[0, \infty): \omega \text { is strictly increasing, onto, continuous and } \omega^{-1}(t) \leq t \leq \omega(t)\right\}
$$

Definition 1 (see [11]). Let $\mathscr{L}$ be a nonempty set. A function $\bar{d}: \mathscr{L} \times \mathscr{L} \longrightarrow[0, \infty)$ is an extended $b$-metric ( $p$-metric, for short) if there exists an $\omega \in \Omega$, such that

(1) $\bar{d}\left(\ell, \ell^{\prime}\right)=0$ iff $\ell=\ell^{\prime}$

(2) $\bar{d}\left(\ell, \ell^{\prime}\right)=\bar{d}\left(\ell^{\prime}, \ell\right)$

(3) $\bar{d}\left(\ell, \ell^{\prime \prime}\right) \leq \omega\left(\bar{d}\left(\ell, \ell^{\prime}\right)+\bar{d}\left(\ell^{\prime}, \ell^{\prime \prime}\right)\right)$

for all $\ell, \ell^{\prime}, \ell^{\prime \prime} \in \mathscr{L}$.

In this case, the pair $(\mathscr{L}, \bar{d})$ is called an extended $b$-metric space or, briefly, a $p$-metric space.

A $b$-metric is a $p$-metric when $\omega(t)=s t$ for some fixed $s \geq 1$, and a metric is a $p$-metric when $\omega(t)=t$. The following example shows that a $p$-metric need not be a $b$-metric.

Example 1 (See [11]). Let $(\mathscr{L}, d)$ be a $b$-metric space with coefficient $s \geq 1$ and let $\bar{d}\left(\ell, \ell^{\prime}\right)=\sinh d\left(\ell, \ell^{\prime}\right)$.

For each $\ell, \ell^{\prime}, \ell^{\prime \prime} \in \mathscr{L}$, we have

$$
\begin{aligned}
\bar{d}\left(\ell, \ell^{\prime \prime}\right) & =\sinh \left(d\left(\ell, \ell^{\prime \prime}\right)\right) \\
& \leq \sinh \left(s d\left(\ell, \ell^{\prime}\right)+s d\left(\ell^{\prime}, \ell^{\prime \prime}\right)\right) \\
& \leq \sinh \left(s \sinh \left(d\left(\ell, \ell^{\prime}\right)\right)+s \sinh \left(d\left(\ell^{\prime}, \ell^{\prime \prime}\right)\right)\right) \\
& =\omega\left(\bar{d}\left(\ell, \ell^{\prime}\right)+\bar{d}\left(\ell^{\prime}, \ell^{\prime \prime}\right)\right) .
\end{aligned}
$$

So, $\bar{d}$ is a $p$-metric with $\omega(t)=\sinh (s \cdot t)$.

Note that $\sinh \left|\ell-\ell^{\prime}\right|$ is not a metric on $\mathbb{R}$, as, e.g.,

$\sinh 5 \approx 74.203 \not 3.627+10.0179 \approx \sinh 2+\sinh 3$.

Similarly, although $d\left(\ell, \ell^{\prime}\right)=\left(\ell-\ell^{\prime}\right)^{2}$ is a $b$-metric on $\mathbb{R}$ with $s=2$, there is no $s \geq 1$, such that $\bar{d}\left(\ell, \ell^{\prime}\right)=\sinh \left(\ell-\ell^{\prime}\right)^{2}$ is a $b$-metric with parameter $s$. Indeed, putting $\ell^{\prime \prime}=0$ and $\ell^{\prime}=1$, we should have $\sinh \ell^{2} \leq s\left(\sinh (\ell-1)^{2}+\sinh 1\right)$ which cannot hold for any fixed $s$ and $\ell$ sufficiently large.

Definition 2 (see [8]). Let $\mathscr{L}$ be a nonempty set. An $S$-metric on $\mathscr{L}$ is a function $S: \mathscr{L}^{3} \longrightarrow[0, \infty)$ so that

(1) $S\left(\ell, \ell^{\prime}, \ell^{\prime \prime}\right) \geq 0$

(2) $S\left(\ell, \ell^{\prime}, \ell^{\prime \prime}\right)=0$ if and only if $\ell=\ell^{\prime}=\ell^{\prime \prime}$

(3) $S\left(\ell, \ell^{\prime}, \ell^{\prime \prime}\right) \leq S(\ell, \ell, a)+S\left(\ell^{\prime}, \ell^{\prime}, a\right)+S\left(\ell^{\prime \prime}, \ell^{\prime \prime}, a\right)$

For all $\ell, \ell^{\prime}, \ell^{\prime \prime}, a \in \mathscr{L}$.

The pair $(\mathscr{L}, S)$ is called an $S$-metric space.
Example 2 (see [8]).

(1) Let $\mathscr{L}=\mathbb{R}^{n}$ and $\|$.$\| be a norm on \mathscr{L}$. Then, $S\left(\ell, \ell^{\prime}, \ell^{\prime \prime}\right)=\left\|\ell^{\prime}+\ell^{\prime \prime}-2 \ell\right\|+\left\|\ell^{\prime}-\ell^{\prime \prime}\right\|$ is an $S$-metric on $\mathscr{L}$.

(2) Let $\mathscr{L}=\mathbb{R}^{n}$ and $\|$.$\| be a norm on \mathscr{L}$. Then, $S\left(\ell, \ell^{\prime}, \ell^{\prime \prime}\right)=\left\|\ell-\ell^{\prime \prime}\right\|+\left\|\ell^{\prime}-\ell^{\prime \prime}\right\|$ is an $S$-metric on $\mathscr{L}$.

(3) Let $\mathscr{L}$ be a nonempty set and $d$ be an ordinary metric on $\mathscr{L}$. Then, $S\left(\ell, \ell^{\prime}, \ell^{\prime \prime}\right)=d\left(\ell, \ell^{\prime}\right)+d\left(\ell^{\prime}, \ell^{\prime \prime}\right)$ is an $S$-metric on $\mathscr{L}$.

Definition 3 (see [17]). Let $\mathscr{L}$ be a nonempty set and $s \geq 1$ be a given real number. Suppose that a mapping $S: \mathscr{L} \times \mathscr{L} \times$ $\mathscr{L} \longrightarrow[0, \infty)$ satisfies

(1) $S\left(\ell, \ell^{\prime}, \ell^{\prime \prime}\right) \geq 0$

(2) $S\left(\ell, \ell^{\prime}, \ell^{\prime \prime}\right)=0$ if and only if $\ell=\ell^{\prime}=\ell^{\prime \prime}$

(3) $S\left(\ell, \ell^{\prime}, \ell^{\prime \prime}\right) \leq s\left(S(\ell, \ell, a)+S\left(\ell^{\prime}, \ell^{\prime}, a\right)+S\left(\ell^{\prime \prime}, \ell^{\prime \prime}, a\right)\right)$

For all $\ell, \ell^{\prime}, \ell^{\prime \prime}, a \in \mathscr{L}$.

Then, $S$ is called a $S_{b}$-metric, and the pair $(\mathscr{L}, S)$ is called a $S_{b}$-metric space.

Definition 4 (see [18]). Let $\mathscr{L}$ be a nonempty set and $\omega \in \Omega$. Suppose that $\bar{s}: \mathscr{L} \times \mathscr{L} \times \mathscr{L} \longrightarrow[0, \infty)$ satisfies

(1) $\bar{s}\left(\ell, \ell^{\prime}, \ell^{\prime \prime}\right) \geq 0$

(2) $\bar{s}\left(\ell, \ell^{\prime}, \ell^{\prime \prime}\right)=0$ if and only if $\ell=\ell^{\prime}=\ell^{\prime \prime}$

(3) $\bar{s}\left(\ell, \ell^{\prime}, \ell^{\prime \prime}\right) \leq \omega\left(\bar{s}(\ell, \ell, a)+\bar{s}\left(\ell^{\prime}, \ell^{\prime}, a\right)+\bar{s}\left(\ell^{\prime \prime}, \ell^{\prime \prime}, a\right)\right)$

For all $\ell, \ell^{\prime}, \ell^{\prime \prime}, a \in \mathscr{L}$.

Then, $\bar{s}$ is called an extended $S$-metric ( $\bar{s}$-metric) and the pair $(\mathscr{L}, \bar{s})$ is called an extended $S$-metric space $(\bar{s}$-metric space).

Each $S$-metric space is an $\bar{s}$-metric space $(\bar{s}$-MS) with $\omega(t)=t$ and every $S_{b}$-MS with parameter $s \geq 1$ is an $\bar{s}$-MS with $\omega(t)=s t$.

Remark 1. In $\bar{s}$-MS, we have $\bar{s}\left(\ell, \ell^{\prime}, \ell^{\prime}\right) \leq \omega\left(2 \bar{s}\left(\ell^{\prime}, \ell^{\prime}, \ell\right)\right)$ and $\bar{s}\left(\ell, \ell, \ell^{\prime}\right) \leq \omega\left(\bar{s}\left(\ell^{\prime}, \ell^{\prime}, \ell\right)\right)$ for all $\ell, \ell^{\prime} \in \mathscr{L}$, i.e, any $\bar{s}$-metric is not symmetry in general.

Proposition 1 (see [18]). Let $(\mathscr{L}, S)$ be $S_{b}-M S$ with coefficient $s \geq 1$ and let $\bar{s}\left(\ell, \ell^{\prime}, \ell^{\prime \prime}\right)=\zeta\left(S\left(\ell, \ell^{\prime}, \ell^{\prime \prime}\right)\right)$, where $\zeta \in \Omega$. Then, $\bar{s}$ is an extended S-metric with $\omega(t)=\zeta(s t)$.

Proof. For all $\ell, \ell^{\prime}, \ell^{\prime \prime}, a \in \mathscr{L}$, 


$$
\begin{aligned}
\bar{s}\left(\ell, \ell^{\prime}, \ell^{\prime \prime}\right) & =\zeta\left(S\left(\ell, \ell^{\prime}, \ell^{\prime \prime}\right)\right) \\
& \leq \zeta\left(s S(\ell, \ell, a)+s S\left(\ell^{\prime}, \ell^{\prime}, a\right)+s S\left(\ell^{\prime \prime}, \ell^{\prime \prime}, a\right)\right) \\
& \leq \zeta\left(s \zeta(S(\ell, \ell, a))+s \zeta\left(S\left(\ell^{\prime}, \ell^{\prime}, a\right)\right)+s \zeta\left(S\left(\ell^{\prime \prime}, \ell^{\prime \prime}, a\right)\right)\right) \\
& =\zeta\left(s \bar{s}(\ell, \ell, a)+s \bar{s}\left(\ell^{\prime}, \ell^{\prime}, a\right)+s \bar{s}\left(\ell^{\prime \prime}, \ell^{\prime \prime}, a\right)\right) \\
& =\omega\left(\bar{s}(\ell, \ell, a)+\bar{s}\left(\ell^{\prime}, \ell^{\prime}, a\right)+\bar{s}\left(\ell^{\prime \prime}, \ell^{\prime \prime}, a\right)\right) .
\end{aligned}
$$

Therefore, $\overline{\mathcal{S}}$ is an extended $S$-metric with $\omega(t)=\zeta(s t)$.

Example 3 (see [18]). Let $(\mathscr{L}, S)$ be $S_{b}$-MS with coefficient $s \geq 1$. Then, $\quad \bar{s}\left(\ell, \ell^{\prime}, \ell^{\prime \prime}\right)=e^{S\left(\ell, \ell^{\prime}, \ell^{\prime \prime}\right)} \sec ^{-1}\left(e^{S\left(\ell, \ell^{\prime}, \ell^{\prime \prime}\right)}\right)$, $\bar{s}\left(\ell, \ell^{\prime}, \ell^{\prime \prime}\right)=\left[S\left(\ell, \ell^{\prime}, \ell^{\prime \prime}\right)+1\right] \sec ^{-1}\left(\left[S\left(\ell, \ell^{\prime}, \ell^{\prime \prime}\right)+1\right]\right)$,

$\bar{s}\left(\ell, \ell^{\prime}, \ell^{\prime \prime}\right)=e^{S\left(\ell, \ell^{\prime}, \ell^{\prime \prime}\right)} \tan ^{-1}\left(e^{S\left(\ell, \ell^{\prime}, \ell^{\prime \prime}\right)}-1\right)$,

$\bar{s}\left(\ell, \ell^{\prime}, \ell^{\prime \prime}\right)=S\left(\ell, \ell^{\prime}, \ell^{\prime \prime}\right) \cosh \left(S\left(\ell, \ell^{\prime}, \ell^{\prime \prime}\right)\right)$

$\bar{s}\left(\ell, \ell^{\prime}, \ell^{\prime \prime}\right)=e^{S\left(\ell, \ell^{\prime}, \ell^{\prime \prime}\right)} \ln \left(1+S\left(\ell, \ell^{\prime}, \ell^{\prime \prime}\right)\right)$,

and

$\bar{s}\left(\ell, \ell^{\prime}, \ell^{\prime \prime}\right)=S\left(\ell, \ell^{\prime}, \ell^{\prime \prime}\right)+\ln \left(1+S\left(\ell, \ell^{\prime}, \ell^{\prime \prime}\right)\right)$ are some

$\bar{s}$-metrics.

Definition 5 (see [18]). Let $(\mathscr{L}, S)$ be $\bar{s}$-MS and $\omega:[0, \infty) \longrightarrow[0, \infty)$. The open ball $B_{\bar{s}}(\ell, r)$ and the closed ball $B_{\bar{s}}[\ell, r]$ with center $\ell$ and radius $r$ are as follows:

$$
\begin{aligned}
& B_{\bar{s}}(\ell, r)=\left\{\ell^{\prime} \in \mathscr{L}: \bar{s}\left(\ell^{\prime}, \ell^{\prime}, \ell\right)<r\right\}, \\
& B_{\bar{s}}[\ell, r]=\left\{\ell^{\prime} \in \mathscr{L}: \bar{s}\left(\ell^{\prime}, \ell^{\prime}, \ell\right) \leq r\right\} .
\end{aligned}
$$

Example 4 (see [18]). Let $\mathscr{L}=\mathbb{R}$. Denote by $\bar{s}\left(\ell, \ell^{\prime}, \ell^{\prime \prime}\right)=$ $\left|\ell^{\prime}+\ell^{\prime \prime}-2 \ell\right|+\left|\ell^{\prime}-\ell^{\prime \prime}\right|$ for all $\ell, \ell^{\prime}, \ell^{\prime \prime} \in \mathbb{R}$ and let $\omega(\ell)=\ell$. Thus,

$$
\begin{aligned}
B_{\bar{s}}(1,2) & =\left\{\ell^{\prime} \in \mathbb{R}: \bar{s}\left(\ell^{\prime}, \ell^{\prime}, 1\right)<2\right\} \\
& =\left\{\ell^{\prime} \in \mathbb{R}:\left|\ell^{\prime}-1\right|<1\right\} \\
& =\left\{\ell^{\prime} \in \mathbb{R}: 0<\ell^{\prime}<2\right\} \\
& =(0,2) .
\end{aligned}
$$

Definition 6 (see [18]). Let $\mathscr{L}$ be $\bar{s}$-MS. A sequence $\left\{\ell_{n}\right\}$ in $\mathscr{L}$ is said to be

(1) $\bar{s}$-Cauchy if, for each $0<\varepsilon$, there exists a positive integer $n_{0}$, such that for all $n, m \geq n_{0}, \bar{s}\left(\ell_{m}, \ell_{n}, \ell_{n}\right)<\varepsilon$

(2) $\bar{s}$-convergent to a point $\ell \in \mathscr{L}$ if, for each $0<\varepsilon$, there exists a positive integer $n_{0}$, such that for all $n \geq n_{0}$, $\bar{s}\left(\ell_{n}, \ell_{n}, \ell\right)<\varepsilon$

(3) An $\bar{s}$-MS $\mathscr{L}$ is called $\bar{s}$-complete, if every $\bar{s}$-Cauchy sequence is $\bar{s}$-convergent in $\mathscr{L}$.

Recently, Eshaghi Gordji et al. [15] introduced the notion of orthogonal sets.

Definition 7 (see [15]). Let $\perp \subseteq \mathscr{L} \times \mathscr{L}$ be a binary relation. If there exists $x_{0} \in \mathscr{L}$, such that $y \perp x_{0}$ for all $y \in \mathscr{L}$ or $x_{0} \perp y$ for all $y \in \mathscr{L}$, then $\mathscr{L}$ is called an orthogonal set (briefly, $O$-set). We denote this $O$-set by $(\mathscr{L}, \perp)$.
Definition 8 (see [15]). Let $(\mathscr{L}, \perp)$ be an $O$-set. A sequence $\left\{\ell_{n}\right\}$ is called an orthogonal sequence (briefly, $O$-sequence) if $x_{n} \perp x_{n+1}$ or $x_{n+1} \perp x_{n}$ for all $n \in \mathbb{N}$.

Definition 9 (see [15]). Let $(\mathscr{L}, \perp, d)$ be an orthogonal set with metric $d$. Then, $\mathscr{L}$ is orthogonally complete (briefly, $\mathrm{O}$-complete) if every Cauchy $\mathrm{O}$-sequence is convergent.

Orthogonal continuous mappings and other concepts can be defined as above.

Motivated by [11], we present the following definitions.

Definition 10. Let $(\mathscr{L}, \bar{s}, \omega, \perp)$ be an $O-\bar{s}$-MS, $\Upsilon$ be a selfmapping on $\mathscr{L}$, and let $\alpha: \mathscr{L} \times \mathscr{L} \times \mathscr{L} \longrightarrow[0,+\infty)$ be a function.

(1) $\Upsilon$ is an $O$ - $\alpha$-admissible mapping if

$$
\ell \perp \ell^{\prime} \perp \ell^{\prime \prime}, \alpha\left(\ell, \ell^{\prime}, \ell^{\prime \prime}\right) \geq 1 \Rightarrow \Upsilon \ell \perp \Upsilon \ell^{\prime} \perp \Upsilon \ell^{\prime \prime}, \alpha\left(\Upsilon \ell, \Upsilon \ell^{\prime}, \Upsilon \ell^{\prime \prime}\right) \geq 1,
$$

for all $\ell, \ell^{\prime}, \ell^{\prime \prime} \in \mathscr{L}$.

(2) The space $(\mathscr{L}, \bar{s}, \omega, \perp)$ is said to be $O$ - $\alpha$-complete if every Cauchy $O$-sequence $\left\{\ell_{n}\right\}$ in $\mathscr{L}$ with $\alpha\left(\ell_{n}, \ell_{n+1}, \ell_{n+2}\right) \geq 1$ for all $n \in \mathbb{N}$ converges in $\mathscr{L}$

(3) $\Upsilon$ is an $O$ - $\alpha$-continuous mapping on $(\mathscr{L}, \bar{s}, \omega, \perp)$, if $\ell_{n} \longrightarrow \ell \quad$ as $n \longrightarrow \infty, \quad \ell_{n} \perp \ell_{n+1} \perp \ell_{n+2}$, and $\alpha\left(\ell_{n}, \ell_{n+1}, \ell_{n+2}\right) \geq 1$ for all $n \in \mathbb{N} \Rightarrow \Upsilon \ell_{n} \longrightarrow \Upsilon \ell$, for given $\ell \in \mathscr{L}$ and arbitrary sequence $\left\{\ell_{n}\right\} \subseteq \mathscr{L}$

(4) $(\mathscr{L}, \bar{s}, \omega, \perp)$ is $O$ - $\alpha$-regular if $\ell_{n} \longrightarrow \ell, \ell_{n} \perp \ell_{n+1} \perp \ell_{n+2}$, and $\alpha\left(\ell_{n}, \ell_{n+1}, \ell_{n+2}\right) \geq 1$ for all $n \in \mathbb{N}$; then, $\alpha\left(\ell_{n}, \ell_{n+1}, \ell\right) \geq 1$ for all $n \in \mathbb{N}$.

Let $\mathscr{L}$ be a nonempty set and $\Gamma: \mathscr{L} \longrightarrow \mathscr{L}$ be a given mapping. For every $\ell \in \mathscr{L}$, let $\Gamma^{-1}(\ell)=\{u \in \mathscr{L}: \Gamma u=\ell\}$.

Motivated by the work in [19], we present the following definition.

Definition 11. Let $\mathscr{L}$ be a set, $\Gamma_{1}, \Gamma_{2}, h: \mathscr{L} \longrightarrow \mathscr{L}$ be mappings such that $\Gamma_{1} \mathscr{L} \cup \Gamma_{2} \mathscr{L} \subseteq h \mathscr{L}$, and let $\alpha: \mathscr{L} \times \mathscr{L} \times \mathscr{L} \longrightarrow[0, \infty)$ be a function. The ordered pair $\left(\Gamma_{1}, \Gamma_{2}\right)$ is said to be

(i) Weakly $O$ - $\alpha$-h-admissible if for all $\ell \in \mathscr{L}, \alpha\left(\Gamma_{1} \ell, \Gamma_{1} \ell, \Gamma_{2} \ell^{\prime}\right) \geq 1$ and $\Gamma_{1} \ell \perp \Gamma_{2} \ell^{\prime}$ for all $\ell^{\prime} \in h^{-1}\left(\Gamma_{1} \ell\right)$, and $\alpha\left(\Gamma_{2} \ell, \Gamma_{2} \ell, \Gamma_{1} \ell^{\prime}\right) \geq 1$ and $\Gamma_{2} \ell \perp \Gamma_{1} \ell^{\prime}$ for all $\ell^{\prime} \in h^{-1}\left(\Gamma_{2} \ell\right)$,

(ii) Partially weakly $O-\alpha$ - $h$-admissible if for all $\ell \in \mathscr{L}$, $\alpha\left(\Gamma_{1} \ell, \Gamma_{1} \ell, \Gamma_{2} \ell^{\prime}\right) \geq 1 \quad$ and $\Gamma_{1} \ell \perp \Gamma_{2} \ell^{\prime} \quad$ for all $\ell^{\prime} \in h^{-1}\left(\Gamma_{1} \ell\right)$ 
(iii) The ordered pair $\left(\Gamma_{1}, \Gamma_{2}\right)$ is said to be rectangular weakly $O-\alpha$ - $h$-admissible (rectangular partially weakly $O$ - $\alpha$-admissible) if it is weakly $O$ - $\alpha$-admissible (partially weakly $O$ - $\alpha$-admissible) with respect to $h$ and $\alpha\left(\ell, \ell, \ell^{\prime \prime}\right) \geq 1, \ell \perp \ell^{\prime \prime}$, $\alpha\left(\ell^{\prime \prime}, \ell^{\prime \prime}, \ell^{\prime}\right) \geq 1$, and $\ell^{\prime \prime} \perp \ell^{\prime}$ imply $\alpha\left(\ell, \ell, \ell^{\prime}\right) \geq 1$ and $\ell \perp \ell^{\prime}$ for all $\ell, \ell^{\prime}, \ell^{\prime \prime} \in \mathscr{L}$.

If, in the previous conditions, $h=I_{\mathscr{L}}$ (the identity mapping), then we can omit the function $h$ in the respective notions.

Definition 12. Let $(\mathscr{L}, \bar{s}, \omega, \perp)$ be an $O-\bar{s}$-MS and $\Gamma_{1}, \Gamma_{2}: \mathscr{L} \longrightarrow \mathscr{L}$. The pair $\left(\Gamma_{1}, \Gamma_{2}\right)$ is said to be
$O$ - $\alpha$-compatible if $\lim _{n \longrightarrow \infty} S\left(\Gamma_{1} \Gamma_{2} \ell_{n}, \Gamma_{1} \Gamma_{2} \ell_{n}, \Gamma_{2} \Gamma_{1} \ell_{n}\right)=0$, whenever $\left\{\ell_{n}\right\}$ is an $O$-sequence in $\mathscr{L}$, such that $\alpha\left(\ell_{n}, \ell_{n}, \ell_{n+1}\right) \geq 1$ for all $n \in \mathbb{N}$ and $\lim _{n \rightarrow \infty} \Gamma_{1} \ell_{n}$ $=\lim _{n \longrightarrow \infty} \Gamma_{2} \ell_{n}=t$ for some $t \in \mathscr{L}$.

In general, an $\bar{s}$-metric mapping $\bar{s}\left(\ell, \ell^{\prime}, \ell^{\prime \prime}\right)$ with nontrivial function $\omega$ need not be jointly continuous in all its variables [18]. Thus, in some proofs, we will need the following simple lemma about the $\bar{s}$-convergent sequences.

Lemma 1. Let a $(\mathscr{L}, \bar{s}, \omega, \perp)$ be $O-\bar{s}-M S$.

(1) Suppose that $\left\{\ell_{n}\right\}$ and $\left\{\ell_{n}^{\prime}\right\}$ are $\bar{s}$-convergent to $\ell$ and $\ell^{\prime}$, respectively. Then, we have

$$
\omega^{-2}\left[\bar{s}\left(\ell, \ell, \ell^{\prime}\right)\right] \leq \liminf _{n \longrightarrow \infty} \bar{s}\left(\ell_{n}, \ell_{n}, \ell_{n}^{\prime}\right) \leq \limsup _{n \rightarrow \infty} \bar{s}\left(\ell_{n}, \ell_{n}, \ell_{n}^{\prime}\right) \leq \omega^{2}\left[\bar{s}\left(\ell, \ell, \ell^{\prime}\right)\right]
$$

In particular, if $\ell=\ell^{\prime}$, then we have $\lim _{n \longrightarrow \infty} \bar{s}\left(\ell_{n}, \ell_{n}^{\prime}, \ell_{n}^{\prime}\right)=0$.
(2) Suppose that $\left\{\ell_{n}\right\}$ is $\bar{s}$-convergent to $\ell$ and $\ell^{\prime \prime} \in \mathscr{L}$ is arbitrary. Then, we have

$$
\omega^{-2}\left[\bar{s}\left(\ell^{\prime \prime}, \ell^{\prime \prime}, \ell\right)\right] \leq \liminf _{n \longrightarrow \infty} \bar{s}\left(\ell^{\prime \prime}, \ell^{\prime \prime}, \ell_{n}\right) \leq \limsup _{n \longrightarrow \infty} \bar{s}\left(\ell^{\prime \prime}, \ell^{\prime \prime}, \ell_{n}\right) \leq \omega\left[2 \bar{s}\left(\ell^{\prime \prime}, \ell^{\prime \prime}, \ell_{n}\right)\right]
$$

(3) Suppose that $\left\{\ell_{n}\right\}$ is $\bar{s}$-convergent to $\ell$ and $\ell^{\prime \prime} \in \mathscr{L}$ is arbitrary. Then, we have

$$
\omega^{-2}\left[\bar{s}\left(\ell, \ell, \ell^{\prime \prime}\right)\right] \leq \liminf _{n \longrightarrow \infty} \bar{s}\left(\ell_{\mathrm{n}}, \ell_{\mathrm{n}}, \ell^{\prime \prime}\right) \leq \limsup _{n \longrightarrow \infty} \overline{\mathrm{s}}\left(\ell_{\mathrm{n}}, \ell_{\mathrm{n}}, \ell^{\prime \prime}\right) \leq \omega^{2}\left[\overline{\mathrm{s}}\left(\ell, \ell, \ell^{\prime \prime}\right)\right] .
$$

Proof. (1) Using the rectangle inequality in the $\bar{s}$-metric space, it is easy to see that

$$
\begin{array}{r}
\bar{s}\left(\ell, \ell, \ell^{\prime}\right) \leq \omega\left[2 \bar{s}\left(\ell, \ell, \ell_{n}\right)+\bar{s}\left(\ell^{\prime}, \ell^{\prime}, \ell_{n}\right)\right] \\
\leq \omega\left[2 \bar{s}\left(\ell, \ell, \ell_{n}\right)+\omega\left[2 \bar{s}\left(\ell^{\prime}, \ell^{\prime}, \ell_{n}^{\prime}\right)+\bar{s}\left(\ell_{n}, \ell_{n}, \ell_{n}^{\prime}\right)\right]\right], \\
\bar{s}\left(\ell_{n}, \ell_{n}, \ell_{n}^{\prime}\right) \leq \omega\left[2 \bar{s}\left(\ell_{n}, \ell_{n}, \ell\right)+\bar{s}\left(\ell_{n}^{\prime}, \ell_{n}^{\prime}, \ell\right)\right] \\
\leq \omega\left[2 \bar{s}\left(\ell_{n}, \ell_{n}, \ell\right)+\omega\left[2 \bar{s}\left(\ell_{n}^{\prime}, \ell_{n}^{\prime}, \ell^{\prime}\right)+\bar{s}\left(\ell, \ell, \ell^{\prime}\right)\right]\right] .
\end{array}
$$

Making $n \longrightarrow \infty$ in the above inequalities, we acquire the desired result.

(2) According to the rectangle inequality, we see that

$$
\begin{aligned}
\bar{s}\left(\ell^{\prime \prime}, \ell^{\prime \prime}, \ell\right) & \leq \omega\left[\bar{s}\left(\ell, \ell, \ell^{\prime \prime}\right)\right] \leq \omega^{2}\left[2 \bar{s}\left(\ell, \ell, \ell_{n}\right)+\bar{s}\left(\ell^{\prime \prime}, \ell^{\prime \prime}, \ell_{n}\right)\right], \\
\bar{s}\left(\ell^{\prime \prime}, \ell^{\prime \prime}, \ell_{n}\right) & \leq \omega\left[2 \bar{s}\left(\ell^{\prime \prime}, \ell^{\prime \prime}, \ell\right)+\bar{s}\left(\ell_{n}, \ell_{n}, \ell\right)\right] .
\end{aligned}
$$

(3) According to the rectangle inequality, we see that 


$$
\begin{aligned}
\bar{s}\left(\ell_{n}, \ell_{n}, \ell^{\prime \prime}\right) & \leq \omega\left[2 \bar{s}\left(\ell_{n}, \ell_{n}, \ell\right)+\bar{s}\left(\ell^{\prime \prime}, \ell^{\prime \prime}, \ell\right)\right] \leq \omega\left[2 \bar{s}\left(\ell_{n}, \ell_{n}, \ell\right)+\omega\left[\bar{s}\left(\ell, \ell, \ell^{\prime \prime}\right)\right]\right] \\
\bar{s}\left(\ell, \ell, \ell^{\prime \prime}\right) & \leq \omega\left[2 \bar{s}\left(\ell, \ell, \ell_{n}\right)+\bar{s}\left(\ell^{\prime \prime}, \ell^{\prime \prime}, \ell_{n}\right)\right] \leq \omega\left[2 \bar{s}\left(\ell, \ell, \ell_{n}\right)+\omega\left[\bar{s}\left(\ell_{n}, \ell_{n}, \ell^{\prime \prime}\right)\right]\right]
\end{aligned}
$$

Recall that the self-mappings $\Gamma_{1}, \Gamma_{2}$ of a set $\mathscr{L}$ are said to be weakly compatible if $\Gamma_{1} \Gamma_{2} \ell=\Gamma_{2} \Gamma_{1} \ell$, whenever $\Gamma_{1} \ell=\Gamma_{2} \ell$ [20].

The function $\omega:[0,+\infty) \longrightarrow[0,+\infty)$ is called an altering distance function (a.d.f) [21], if

(1) $\Phi$ is continuous and nondecreasing

(2) $₫(t)=0$ iff $t=0$

\section{Main Results}

Let $(\mathscr{L}, \bar{s}, \omega, \perp)$ be $O-\bar{s}$-MS, and let $\Gamma_{1}, \Gamma_{2}, \Upsilon_{1}, \Upsilon_{2}: \mathscr{L} \longrightarrow \mathscr{L}$ be four self-mappings. Throughout this study, unless otherwise stated, for all $\ell, \ell^{\prime} \in \mathscr{L}, M\left(\ell, \ell, \ell^{\prime}\right)$ will denote an arbitrary element of the set

$$
\begin{array}{r}
\left\{\bar{s}\left(\Upsilon_{2} \ell, \Upsilon_{2} \ell, \Upsilon_{1} \ell^{\prime}\right), \omega^{-1}\left[\frac{\bar{s}\left(\Upsilon_{2} \ell, \Upsilon_{2} \ell, \Gamma_{1} \ell\right)+\bar{s}\left(\Upsilon_{1} \ell^{\prime}, \Upsilon_{1} \ell^{\prime}, \Gamma_{2} \ell^{\prime}\right)}{2}\right]\right. \\
\left.\omega^{-1}\left[\frac{\bar{s}\left(\Upsilon_{2} \ell, \Upsilon_{2} \ell, \Gamma_{2} \ell^{\prime}\right)+\bar{s}\left(\Upsilon_{1} \ell^{\prime}, \Upsilon_{1} \ell^{\prime}, \Gamma_{1} \ell\right)}{2}\right]\right\} .
\end{array}
$$

Theorem 1. Let $(\mathscr{L}, \bar{s}, \omega, \perp)$ be an $O$ - $\alpha$-complete $O-\bar{s}-M S$, $\alpha: \mathscr{L} \times \mathscr{L} \times \mathscr{L} \longrightarrow[0, \infty)$, and let $\Gamma_{1}, \Gamma_{2}, \Upsilon_{1}, \Upsilon_{2}: \mathscr{L} \longrightarrow \mathscr{L}$ be such that $\Gamma_{1}(\mathscr{L}) \subseteq \Upsilon_{1}(\mathscr{L})$ and $\Gamma_{2}(\mathscr{L}) \subseteq \Upsilon_{2}(\mathscr{L})$.

Suppose that for all $\ell, \ell^{\prime} \in \mathscr{L}$ with $\alpha\left(\Upsilon_{1} \ell, \Upsilon_{1} \ell, \Upsilon_{2} \ell^{\prime}\right) \geq 1$ and $\Upsilon_{1} \ell \perp \Upsilon_{2} \ell^{\prime}$,

$$
\pi\left(\omega\left(\bar{s}\left(\Gamma_{1} \ell, \Gamma_{1} \ell, \Gamma_{2} \ell^{\prime}\right)\right)\right) \leq \pi\left(M\left(\ell, \ell, \ell^{\prime}\right)\right)-\varpi\left(M\left(\ell, \ell, \ell^{\prime}\right)\right),
$$

where $\pi, \emptyset$ are two a.d.f. Assume that $\Gamma_{1}, \Gamma_{2}, \Upsilon_{1}$, and $\Upsilon_{2}$ are $O-\alpha$-continuous, the pairs $\left(\Gamma_{1}, \Upsilon_{2}\right)$ and $\left(\Gamma_{2}, \Upsilon_{1}\right)$ are $O$ - $\alpha$-compatible, and the pairs $\left(\Gamma_{1}, \Upsilon_{2}\right)$ and $\left(\Gamma_{2}, \Upsilon_{1}\right)$ are rectangular partially weakly O- $\alpha-\Upsilon_{1}$-admissible and $\mathrm{O}-\alpha-\Upsilon_{2}$-admissible, respectively. Then, the pairs $\left(\Gamma_{1}, \Upsilon_{2}\right)$ and $\left(\Gamma_{2}, \Upsilon_{1}\right)$ have a coincidence point $\ell^{\prime \prime}$ in $\mathscr{L}$. Moreover, if $\alpha\left(\Upsilon_{2} \ell^{\prime \prime}, \Upsilon_{2} \ell^{\prime \prime}, \Upsilon_{1} \ell^{\prime \prime}\right) \geq 1$ and $\Upsilon_{2} \ell^{\prime \prime} \perp \Upsilon_{1} \ell^{\prime \prime}$, then $\ell^{\prime \prime}$ is a coincidence point of $\Gamma_{1}, \Gamma_{2}, \Upsilon_{1}$, and $\Upsilon_{2}$.

Proof. Let $\ell_{0} \in \mathscr{L}$. Choose $\ell_{1} \in \mathscr{L}$, such that $\Gamma_{1} \ell_{0}=\Upsilon_{1} \ell_{1}$, and $\ell_{2} \in \mathscr{L}$, such that $\Gamma_{2} \ell_{1}=\Upsilon_{2} \ell_{2}$. Continuing in this way, we construct a sequence $\left\{\ell_{n}^{\prime \prime}\right\}$ defined by

$$
\begin{aligned}
& \ell_{2 n+1}^{\prime \prime}=\Upsilon_{1} \ell_{2 n+1}=\Gamma_{1} \ell_{2 n}, \\
& \ell_{2 n+2}^{\prime \prime}=\Upsilon_{2} \ell_{2 n+2}=\Gamma_{2} \ell_{2 n+1},
\end{aligned}
$$

for all $n \geq 0$. As $\ell_{1} \in \Upsilon_{1}^{-1}\left(\Gamma_{1} \ell_{0}\right)$ and $\ell_{2} \in \Upsilon_{2}^{-1}\left(\Gamma_{2} \ell_{1}\right)$ and the pairs $\left(\Gamma_{1}, \Gamma_{2}\right)$ and $\left(\Gamma_{2}, \Gamma_{1}\right)$ are partially weakly
$O-\alpha-\Upsilon_{1}$-admissible and $O-\alpha-\Upsilon_{2}$-admissible, respectively, we have

$$
\begin{aligned}
\alpha\left(\Upsilon_{1} \ell_{1}, \Upsilon_{1} \ell_{1}, \Upsilon_{2} \ell_{2}\right) & =\alpha\left(\Gamma_{1} \ell_{0}, \Gamma_{1} \ell_{0}, \Gamma_{2} \ell_{1}\right) \geq 1 \\
& \Upsilon_{1} \ell_{1} \perp \Upsilon_{2} \ell_{2}\left(\Gamma_{1} \ell_{0} \perp \Gamma_{2} \ell_{1}\right), \\
\alpha\left(\Upsilon_{2} \ell_{2}, \Upsilon_{2} \ell_{2}, \Upsilon_{1} \ell_{3}\right) & =\alpha\left(\Gamma_{2} \ell_{1}, \Gamma_{2} \ell_{1}, \Gamma_{1} \ell_{2}\right) \geq 1 \\
& \Upsilon_{2} \ell_{2} \perp \Upsilon_{1} \ell_{3}\left(\Gamma_{2} \ell_{1} \perp \Gamma_{1} \ell_{2}\right) .
\end{aligned}
$$

Continuing this way, we acquire that

$$
\begin{gathered}
\alpha\left(\Upsilon_{1} \ell_{2 n+1}, \Upsilon_{1} \ell_{2 n+1}, \Upsilon_{2} \ell_{2 n+2}\right)=\alpha\left(\ell_{2 n+1}^{\prime \prime}, \ell_{2 n+1}^{\prime \prime}, \ell_{2 n+2}^{\prime \prime}\right) \geq 1 \\
\Upsilon_{1} \ell_{2 n+1} \perp \Upsilon_{2} \ell_{2 n+2}\left(\ell_{2 n+1}^{\prime \prime} \perp \ell_{2 n+2}^{\prime \prime}\right), \\
\alpha\left(\Upsilon_{2} \ell_{2 n+2}, \Upsilon_{2} \ell_{2 n+2}, \Upsilon_{1} \ell_{2 n+3}\right)=\alpha\left(\ell_{2 n+2}^{\prime \prime}, \ell_{2 n+2}^{\prime \prime}, \ell_{2 n+3}^{\prime \prime}\right) \geq 1, \\
\Upsilon_{2} \ell_{2 n+2} \perp \Upsilon_{1} \ell_{2 n+3}\left(\ell_{2 n+2}^{\prime \prime} \perp \ell_{2 n+3}^{\prime \prime}\right),
\end{gathered}
$$

for all $n \geq 0$.

Three steps will be taken to finish the proof.

Step 1. We will prove that $\lim _{k \rightarrow \infty} \bar{s}\left(\ell_{k}^{\prime \prime}, \ell_{k}^{\prime \prime}, \ell_{k+1}^{\prime \prime}\right)=0$. Let $\bar{s}_{k}=\bar{s}\left(\ell_{k_{1 \prime}^{\prime \prime}}^{\prime \prime}, \ell_{k}^{\prime \prime}, \ell_{k+1}^{\prime \prime}\right)$. Suppose that $\bar{s}_{k_{0}}=0$ for some $k_{0}$. Then, $\ell_{k_{0}}^{\prime \prime}=\ell_{k_{0}+1}^{\prime \prime}$.

If $k_{0}=2 n$, then $\ell_{2 n}^{\prime \prime}=\ell_{2 n+1}^{\prime \prime}$ gives $\ell_{2 n+1}^{\prime \prime}=\ell_{2 n+2}^{\prime \prime}$. Indeed, 


$$
\begin{aligned}
\pi\left(\omega\left(\bar{s}\left(\ell_{2 n+1}^{\prime \prime}, \ell_{2 n+1}^{\prime \prime}, \ell_{2 n+2}^{\prime \prime}\right)\right)\right) & =\pi\left(\omega\left(\bar{s}\left(\Gamma_{1} \ell_{2 n}, \Gamma_{1} \ell_{2 n}, \Gamma_{2} \ell_{2 n+1}\right)\right)\right) \\
& \leq \pi\left(M\left(\ell_{2 n}, \ell_{2 n}, \ell_{2 n+1}\right)\right)-\omega\left(M\left(\ell_{2 n}, \ell_{2 n}, \ell_{2 n+1}\right)\right)
\end{aligned}
$$

where

$$
\begin{aligned}
& M\left(\ell_{2 n}, \ell_{2 n}, \ell_{2 n+1}\right) \\
& \in\left\{\bar{s}\left(\Upsilon_{2} \ell_{2 n}, \Upsilon_{2} \ell_{2 n}, \Upsilon_{1} \ell_{2 n+1}\right), \omega^{-1}\left[\frac{\bar{s}\left(\Upsilon_{2} \ell_{2 n}, \Upsilon_{2} \ell_{2 n}, \Gamma_{1} \ell_{2 n}\right)+\bar{s}\left(\Upsilon_{1} \ell_{2 n+1}, \Upsilon_{1} \ell_{2 n+1}, \Gamma_{2} \ell_{2 n+1}\right)}{2}\right],\right. \\
& \left.\omega^{-1}\left[\frac{\bar{s}\left(\Upsilon_{2} \ell_{2 n}, \Upsilon_{2} \ell_{2 n}, \Gamma_{2} \ell_{2 n+1}\right)+\bar{s}\left(\Upsilon_{1} \ell_{2 n+1}, \Upsilon_{1} \ell_{2 n+1}, \Gamma_{1} \ell_{2 n}\right)}{2}\right]\right\} \\
& =\left\{\bar{s}\left(\ell_{2 n}^{\prime \prime}, \ell_{2 n}^{\prime \prime}, \ell_{2 n+1}^{\prime \prime}\right), \omega^{-1}\left[\frac{\bar{s}\left(\ell_{2 n}^{\prime \prime}, \ell_{2 n}^{\prime \prime}, \ell_{2 n+1}^{\prime \prime}\right)+\bar{s}\left(\ell_{2 n+1}^{\prime \prime}, \ell_{2 n+1}^{\prime \prime}, \ell_{2 n+2}^{\prime \prime}\right)}{2}\right],\right. \\
& \left.\omega^{-1}\left[\frac{\bar{s}\left(\ell_{2 n}^{\prime \prime}, \ell_{2 n}^{\prime \prime}, \ell_{2 n+2}^{\prime \prime}\right)+\bar{s}^{\prime \prime}\left(\ell_{2 n+1}^{\prime \prime}, \ell_{2 n+1}^{\prime \prime}, \ell_{2 n+2}^{\prime \prime}\right)}{2}\right]\right\}^{\prime \prime} \\
& =\left\{0, \omega^{-1}\left[\frac{\bar{s}\left(\ell_{2 n+1}^{\prime \prime}, \ell_{2 n+1}^{\prime \prime}, \ell_{2 n+2}^{\prime \prime}\right)}{2}\right], \omega^{-1}\left[\frac{\bar{s}\left(\ell_{2 n}^{\prime \prime}, \ell_{2 n}^{\prime \prime}, \ell_{2 n+2}^{\prime \prime}\right)}{2}\right]\right\} .
\end{aligned}
$$

If $M\left(\ell_{2 n}, \ell_{2 n}, \ell_{2 n+1}\right)=\omega^{-1}\left[\bar{s}\left(\ell_{2 n+1}^{\prime \prime}, \ell_{2 n+1}^{\prime \prime}, \ell_{2 n+2}^{\prime \prime}\right) / 2\right]$, then (19) becomes

$$
\begin{aligned}
\pi\left(\omega\left(\bar{s}\left(\ell_{2 n+1}^{\prime \prime}, \ell_{2 n+1}^{\prime \prime}, \ell_{2 n+2}^{\prime \prime}\right)\right)\right) & \leq \pi\left(\omega^{-1}\left(\frac{\bar{s}\left(\ell_{2 n+1}^{\prime \prime}, \ell_{2 n+1}^{\prime \prime}, \ell_{2 n+2}^{\prime \prime}\right)}{2}\right)\right)-\omega\left(\omega^{-1}\left[\bar{s} \frac{\left(\ell_{2 n+1}^{\prime \prime}, \ell_{2 n+1}^{\prime \prime}, \ell_{2 n+2}^{\prime \prime}\right)}{2}\right)\right. \\
& \leq \pi\left(\omega\left(\bar{s}\left(\ell_{2 n+1}^{\prime \prime}, \ell_{2 n+1}^{\prime \prime}, \ell_{2 n+2}^{\prime \prime}\right)\right)-\omega\left(\omega^{-1}\left[\frac{\bar{s}\left(\ell_{2 n+1}^{\prime \prime}, \ell_{2 n+1}^{\prime \prime}, \ell_{2 n+2}^{\prime \prime}\right)}{2}\right]\right),\right.
\end{aligned}
$$

which implies that $\Phi\left(\omega^{-1}\left[\bar{s}\left(\ell_{2 n+1}^{\prime \prime}, \ell_{2 n+1}^{\prime \prime}, \ell_{2 n+2}{ }^{\prime \prime}\right) / 2\right]\right)=0$, that is, $\ell_{2 n}^{\prime \prime}=\ell_{2 n+1}^{\prime \prime}=\ell_{2 n+2}^{\prime \prime}$.
If $M\left(\ell_{2 n}, \ell_{2 n}, \ell_{2 n+1}\right)=\omega^{-1}\left[\bar{s}\left(\ell_{2 n}^{\prime \prime}, \ell_{2 n}^{\prime \prime}, \ell_{2 n+2}^{\prime \prime}\right) / 2\right]$, then (19) becomes

$$
\begin{aligned}
\pi\left(\omega\left(\bar{s}\left(\ell_{2 n+1}^{\prime \prime}, \ell_{2 n+1}^{\prime \prime}, \ell_{2 n+2}^{\prime \prime}\right)\right)\right) & \leq \pi\left(\omega^{-1}\left(\frac{\bar{s}\left(\ell_{2 n}^{\prime \prime}, \ell_{2 n}^{\prime \prime}, \ell_{2 n+2}^{\prime \prime}\right)}{2}\right)\right)-\omega\left(\omega^{-1}\left[\frac{\bar{s}\left(\ell_{2 n}^{\prime \prime}, \ell_{2 n}^{\prime \prime}, \ell_{2 n+2}^{\prime \prime}\right)}{2}\right]\right) \\
& \leq \pi\left(\bar{s}\left(\ell_{2 n+1}^{\prime \prime}, \ell_{2 n+1}^{\prime \prime}, \ell_{2 n+2}^{\prime \prime}\right)\right)-\omega\left(\omega^{-1}\left[\frac{\bar{s}\left(\ell_{2 n}^{\prime \prime}, \ell_{2 n}^{\prime \prime}, \ell_{2 n+2}^{\prime \prime}\right)}{2}\right]\right),
\end{aligned}
$$

which implies that $\omega\left(\omega^{-1}\left[\bar{s}\left(\ell_{2 n+1}^{\prime \prime}, \ell_{2 n+1}^{\prime \prime}, \ell_{2 n+2}{ }^{\prime \prime}\right) / 2\right]\right)=0$, that

$$
\bar{s}_{k}=\bar{s}\left(\ell_{k}^{\prime \prime}, \ell_{k}^{\prime \prime}, \ell_{k+1}^{\prime \prime}\right)>0,
$$

is, $\ell_{2 n}^{\prime \prime}=\ell_{2 n+1}^{\prime \prime}=\ell_{2 n+2}^{\prime \prime}$

Similarly, if $k_{0}=2 n+1$, then $\ell_{2 n+1}^{\prime \prime}=\ell_{2 n+2}^{\prime \prime}$ gives $\ell_{2 n+2}^{\prime \prime}=\ell_{2 n+3}$. Continuing this process, we find that $\ell_{k}^{\prime \prime}$ is a constant sequence for $k \geq k_{0}$. Hence, $\lim _{k \rightarrow \infty} \bar{s}\left(\ell_{k}^{\prime \prime}, \ell_{k}^{\prime \prime}, \ell_{k+1}^{\prime \prime}\right)=0$ is correct.

Now, suppose that for all $k$. We show that

$$
\bar{s}\left(\ell_{k}^{\prime \prime}, \ell_{k}^{\prime \prime}, \ell_{k+1}^{\prime \prime}\right) \leq \bar{s}\left(\ell_{k}^{\prime \prime}, \ell_{k}^{\prime \prime}, \ell_{k+1}^{\prime \prime}\right),
$$

for each $k=1,2,3, \cdots$. 
Let $k=2 n$ and $\bar{s}\left(\ell_{2 n+1}^{\prime \prime}, \ell_{2 n+1}^{\prime \prime}, \ell_{2 n+2}^{\prime \prime}\right) \geq \bar{s}\left(\ell_{2 n}^{\prime \prime}, \ell_{2 n}^{\prime \prime}, \ell_{2 n+1}^{\prime \prime}\right) \quad$ where $>0$, for $n \geq 0$. Then, as $\alpha\left(\Upsilon_{2} \ell_{2 n}, \Upsilon_{2} \ell_{2 n}, \Upsilon_{1} \ell_{2 n+1}\right) \geq 1$ and $\Upsilon_{2} \ell_{2 n} \perp \Upsilon_{1} \ell_{2 n+1}$, using (15), we acquire that

$$
\begin{array}{r}
\pi\left(\omega\left(\bar{s}\left(\ell_{2 n+1}^{\prime \prime}, \ell_{2 n+1}^{\prime \prime}, \ell_{2 n+2}^{\prime \prime}\right)\right)\right)=\pi\left(\omega\left(\bar{s}\left(\Gamma_{1} \ell_{2 n}, \Gamma_{1} \ell_{2 n}, \Gamma_{2} \ell_{2 n+1}\right)\right)\right) \\
\leq \pi\left(M\left(\ell_{2 n}, \ell_{2 n}, \ell_{2 n+1}\right)\right)-\Phi\left(M\left(\ell_{2 n}, \ell_{2 n}, \ell_{2 n+1}\right)\right),
\end{array}
$$

$$
\begin{aligned}
& M\left(\ell_{2 n}, \ell_{2 n}, \ell_{2 n+1}\right) \\
& \in\left\{\bar{s}\left(\Upsilon_{2} \ell_{2 n}, \Upsilon_{2} \ell_{2 n}, \Upsilon_{1} \ell_{2 n+1}\right), \omega^{-1}\left[\frac{\bar{s}\left(\Upsilon_{2} \ell_{2 n}, \Upsilon_{2} \ell_{2 n}, \Gamma_{1} \ell_{2 n}\right)+\bar{s}\left(\Upsilon_{1} \ell_{2 n+1}, \Upsilon_{1} \ell_{2 n+1}, \Gamma_{2} \ell_{2 n+1}\right)}{2}\right],\right. \\
& \left.\omega^{-1}\left[\frac{\bar{s}\left(\Upsilon_{2} \ell_{2 n}, \Upsilon_{2} \ell_{2 n}, \Gamma_{2} \ell_{2 n+1}\right)+\bar{s}\left(\Upsilon_{1} \ell_{2 n+1}, \Upsilon_{1} \ell_{2 n+1}, \Upsilon_{1} \ell_{2 n}\right)}{2}\right]\right\} \\
& =\left\{\bar{s}\left(\ell_{2 n}^{\prime \prime}, \ell_{2 n}^{\prime \prime}, \ell_{2 n+1}^{\prime \prime}\right), \omega^{-1}\left[\frac{\bar{s}\left(\ell_{2 n}^{\prime \prime}, \ell_{2 n}^{\prime \prime}, \ell_{2 n+1}^{\prime \prime}\right)+\bar{s}\left(\ell_{2 n+1}^{\prime \prime}, \ell_{2 n+1}^{\prime \prime}, \ell_{2 n+2}^{\prime \prime}\right)}{2}\right],\right. \\
& \left.\omega^{-1}\left[\frac{\bar{s}\left(\ell_{2 n}^{\prime \prime}, \ell_{2 n}^{\prime \prime}, \ell_{2 n+2}^{\prime \prime}\right)}{2}\right]\right\} .
\end{aligned}
$$

If

$$
\begin{aligned}
M\left(\ell_{2 n}, \ell_{2 n}, \ell_{2 n+1}\right) & =\omega^{-1}\left[\frac{\bar{s}\left(\ell_{2 n}^{\prime \prime}, \ell_{2 n}^{\prime \prime}, \ell_{2 n+1}^{\prime \prime}\right)+\bar{s}\left(\ell_{2 n+1}^{\prime \prime}, \ell_{2 n+1}^{\prime \prime}, \ell_{2 n+2}^{\prime \prime}\right)}{2}\right] \\
& \leq \omega^{-1}\left(\bar{s}\left(\ell_{2 n+1}^{\prime \prime}, \ell_{2 n+1}^{\prime \prime}, \ell_{2 n+2}^{\prime \prime}\right)\right),
\end{aligned}
$$

As $\bar{s}\left(\ell_{2 n+1}^{\prime \prime}, \ell_{2 n+1}^{\prime \prime}, \ell_{2 n+2}^{\prime \prime}\right) \geq \bar{s}\left(\ell_{2 n}^{\prime \prime}, \ell_{2 n}^{\prime \prime}, \ell_{2 n+1}^{\prime \prime}\right)$, then from (25), we have

$$
\begin{aligned}
\pi\left(\omega\left(\bar{s}\left(\ell_{2 n+1}^{\prime \prime}, \ell_{2 n+1}^{\prime \prime}, \ell_{2 n+2}^{\prime \prime}\right)\right)\right) & \leq \pi\left(\omega^{-1}\left(\frac{\bar{s}\left(\ell_{2 n}^{\prime \prime}, \ell_{2 n}^{\prime \prime}, \ell_{2 n+1}^{\prime \prime}\right)+\bar{s}\left(\ell_{2 n+1}^{\prime \prime}, \ell_{2 n+1}^{\prime \prime}, \ell_{2 n+2}^{\prime \prime}\right)}{2}\right)\right) \\
& -\omega\left(\omega^{-1}\left[\frac{\bar{s}\left(\ell_{2 n+1}^{\prime \prime}, \ell_{2 n+1}^{\prime \prime}, \ell_{2 n+2}^{\prime \prime}\right)+\bar{s}\left(\ell_{2 n+1}^{\prime \prime}, \ell_{2 n+1}^{\prime \prime}, \ell_{2 n+2}^{\prime \prime}\right)}{2}\right]\right) \\
& \leq \pi\left(\omega\left(\bar{s}\left(\ell_{2 n+1}^{\prime \prime}, \ell_{2 n+1}^{\prime \prime}, \ell_{2 n+2}^{\prime \prime}\right)\right)\right)-\omega\left(\omega^{-1}\left[\frac{\bar{s}\left(\ell_{2 n+1}^{\prime \prime}, \ell_{2 n+1}^{\prime \prime}, \ell_{2 n+2}^{\prime \prime}\right)}{2}\right]\right),
\end{aligned}
$$


which implies that $\Phi\left(\omega^{-1}\left[\bar{s}\left(\ell_{2 n}^{\prime \prime}, \ell_{2 n}^{\prime \prime}, \ell_{2 n+1}^{\prime \prime}\right)+\bar{s}\left(\ell_{2 n+1}^{\prime \prime}, \ell_{2 n+1}^{\prime \prime}\right.\right.\right.$, $\left.\left.\left.\ell_{2 n+2}^{\prime \prime}\right) / 2\right]\right) \leq 0$. This is possible only if $\left[\bar{s}\left(\ell_{2 n}^{\prime \prime}, \ell_{2 n}, \ell_{2 n+1}^{\prime \prime}\right)\right.$ $\left.+\bar{s}\left(\ell_{2 n+1}^{\prime \prime}, \ell_{2 n+1}^{\prime \prime}, \ell_{2 n+2}^{\prime \prime}\right) / 2\right]=0$, that is, $\bar{s}\left(\ell_{2 n}^{\prime \prime}, \ell_{2 n}, \ell_{2 n+1}^{\prime \prime}\right)=0$, a contradiction to (23). Hence, $\bar{s}\left(\ell_{2 n+1}^{\prime \prime}, \ell_{2 n+1}^{\prime \prime}, \ell_{2 n+2}^{\prime \prime}\right)$ $\leq \bar{s}\left(\ell_{2 n}^{\prime \prime}, \ell_{2 n}^{\prime \prime}, \ell_{2 n+1}^{\prime \prime}\right)$ for all $n \geq 0$.

Therefore, (24) is proved for $k=2 n$.

Analogously,

$$
\bar{s}\left(\ell_{2 n+2}^{\prime \prime}, \ell_{2 n+2}^{\prime \prime}, \ell_{2 n+3}^{\prime \prime}\right) \leq \bar{s}\left(\ell_{2 n+1}^{\prime \prime}, \ell_{2 n+1}^{\prime \prime}, \ell_{2 n+2}^{\prime \prime}\right),
$$

for all $n \geq 0$.

Similarly, for other values of $M\left(\ell_{2 n}, \ell_{2 n}, \ell_{2 n+1}\right)$, we can see that $\left\{\bar{s}\left(\ell_{k}^{\prime \prime}, \ell_{k}^{\prime \prime}, \ell_{k+1}^{\prime \prime}\right)\right\}$ is a nondecreasing sequence of nonnegative real numbers. Therefore,

$$
\lim _{k \longrightarrow \infty} \bar{s}\left(\ell_{k}^{\prime \prime}, \ell_{k}^{\prime \prime}, \ell_{k+1}^{\prime \prime}\right)=r,
$$

for some $r \geq 0$.

We know that

$$
\begin{aligned}
& M\left(\ell_{2 n}, \ell_{2 n}, \ell_{2 n+1}\right) \in\left\{\bar{s}\left(\ell_{2 n}^{\prime \prime}, \ell_{2 n}^{\prime \prime}, \ell_{2 n+1}^{\prime \prime}\right), \omega^{-1}\left[\frac{\bar{s}\left(\ell_{2 n}^{\prime \prime}, \ell_{2 n}^{\prime \prime}, \ell_{2 n+1}^{\prime \prime}\right)+\bar{s}\left(\ell_{2 n+1}^{\prime \prime}, \ell_{2 n+1}^{\prime \prime}, \ell_{2 n+2}^{\prime \prime}\right)}{2}\right],\right. \\
&\left.\omega^{-1}\left[\frac{\bar{s}\left(\ell_{2 n}^{\prime \prime}, \ell_{2 n}^{\prime \prime}, \ell_{2 n+2}^{\prime \prime}\right)+\bar{s}\left(\ell_{2 n+1}^{\prime \prime}, \ell_{2 n+1}^{\prime \prime}, \ell_{2 n+1}^{\prime \prime}\right)}{2}\right]\right\} .
\end{aligned}
$$

Substituting possible values of $M\left(\ell_{2 n}, \ell_{2 n}, \ell_{2 n+1}\right)$ in (25) and making $n \longrightarrow \infty$, we acquire that $r=0$. For instance, let

$$
M\left(\ell_{2 n}, \ell_{2 n}, \ell_{2 n+1}\right)=\omega^{-1}\left[\frac{\bar{s}\left(\ell_{2 n}^{\prime \prime}, \ell_{2 n}^{\prime \prime}, \ell_{2 n+2}^{\prime \prime}\right)+\bar{s}\left(\ell_{2 n+1}^{\prime \prime}, \ell_{2 n+1}^{\prime \prime}, \ell_{2 n+1}^{\prime \prime}\right)}{2}\right] .
$$

Then, from (25), we have

$$
\begin{aligned}
& \pi\left(\omega\left(\bar{s}\left(\ell_{2 n+1}^{\prime \prime}, \ell_{2 n+1}^{\prime \prime}, \ell_{2 n+2}^{\prime \prime}\right)\right)\right) \\
& \leq \pi\left(\omega^{-1}\left(\frac{\bar{s}\left(\ell_{2 n+1}^{\prime \prime}, \ell_{2 n+1}^{\prime \prime}, \ell_{2 n+2}^{\prime \prime}\right)}{2}\right)\right)-\omega\left(\omega^{-1}\left[\frac{\bar{s}\left(\ell_{2 n+1}^{\prime \prime}, \ell_{2 n+1}^{\prime \prime}, \ell_{2 n+2}^{\prime \prime}\right)}{2}\right]\right) \\
& \leq \pi\left(\frac{\bar{s}\left(\ell_{2 n}^{\prime \prime}, \ell_{2 n}^{\prime \prime}, \ell_{2 n+2}^{\prime \prime}\right)+\bar{s}\left(\ell_{2 n+1}^{\prime \prime}, \ell_{2 n+1}^{\prime \prime}, \ell_{2 n+2}^{\prime \prime}\right)}{2}\right)-\omega\left(\omega^{-1}\left[\frac{\bar{s}\left(\ell_{2 n+1}^{\prime \prime}, \ell_{2 n+1}^{\prime \prime}, \ell_{2 n+2}^{\prime \prime}\right)}{2}\right]\right) \\
& \text { in (33), using (30) and the continuity of } \quad r=\lim _{k \rightarrow \infty} \bar{s}\left(\ell_{k}^{\prime \prime}, \ell_{k}^{\prime \prime}, \ell_{k+1}^{\prime \prime}\right)=\lim _{n \rightarrow \infty} \bar{s}\left(\ell_{2 n}^{\prime \prime}, \ell_{2 n}^{\prime \prime}, \ell_{2 n+1}^{\prime \prime}\right)=0
\end{aligned}
$$

Letting $n \longrightarrow \infty$ in (33), using (30) and the continuity of

$$
Ф\left(\lim _{n \longrightarrow \infty} \omega^{-1}\left[\frac{\bar{s}\left(\ell_{2 n}^{\prime \prime}, \ell_{2 n}^{\prime \prime}, \ell_{2 n+2}^{\prime \prime}\right)}{2}\right]\right)=0
$$

Hence, $\lim _{n \longrightarrow \infty} \omega^{-1}\left[\bar{s}\left(\ell_{2 n}^{\prime \prime}, \ell_{2 n}^{\prime \prime}, \ell_{2 n+2}^{\prime \prime}\right) / 2\right]=0$.

Now, taking into account (33) and letting $n \longrightarrow \infty$, we find that $\pi(\omega(r)) \leq \pi(0)-\Phi(0)$. Hence, $r=0$. Similarly, for the other values of $M\left(\ell_{2 n}, \ell_{2 n}, \ell_{2 n+1}\right)$, we can show that

Step 2. We will show the $\bar{s}$-Cauchyness of $\left\{\ell_{n}^{\prime \prime}\right\}$ in $\mathscr{L}$. Assume that for some $\varepsilon>0$, we have subsequences $\left\{\ell_{2 m(k)}^{\prime \prime}\right\},\left\{\ell_{2 n(k)}^{\prime \prime}\right\} \subseteq\left\{\ell_{2 n}^{\prime \prime}\right\}$, such that $n(k)>m(k) \geq k$ and

$$
\bar{s}\left(\ell_{2 m(k)}^{\prime \prime}, \ell_{2 m(k)}^{\prime \prime}, \ell_{2 n(k)}^{\prime \prime}\right) \geq \varepsilon,
$$

in which $n(k)$ is the least index, i.e., 


$$
\bar{s}\left(\ell_{2 m(k)}^{\prime \prime}, \ell_{2 m(k)}^{\prime \prime}, \ell_{2 n(k)-1}^{\prime \prime}\right)<\varepsilon
$$

From the rectangle inequality and (36) and (37), we have

$$
\begin{aligned}
& \varepsilon \leq \bar{s}\left(\ell_{2 m(k)}^{\prime \prime}, \ell_{2 m(k)}^{\prime \prime}, \ell_{2 n(k)}^{\prime \prime}\right) \\
& \leq \omega\left[\bar{s}\left(\ell_{2 m(k)}^{\prime \prime}, \ell_{2 m(k)}^{\prime \prime}, \ell_{2 n(k)-1}^{\prime \prime}\right)+\bar{s}\left(\ell_{2 m(k)}^{\prime \prime}, \ell_{2 m(k)}^{\prime \prime}, \ell_{2 n(k)-1}^{\prime \prime}\right)+\bar{s}\left(\ell_{2 m(k)}^{\prime \prime}, \ell_{2 m(k)}^{\prime \prime}, \ell_{2 n(k)-1}^{\prime \prime}\right)\right] . \\
& \text { mit as } k \longrightarrow \infty \text { in (38), from (35), we } \quad \underset{k \rightarrow \infty}{\limsup } \bar{s}\left(\ell_{2 m(k)}^{\prime \prime}, \ell_{2 m(k)}^{\prime \prime}, \ell_{2 n(k)}^{\prime}\right) \leq \omega(\varepsilon) .
\end{aligned}
$$

Taking the limit as $k \longrightarrow \infty$ in (38), from (35), we acquire that

Using the rectangle inequality again, we have

$$
\begin{aligned}
& \bar{s}\left(\ell_{2 m(k)}^{\prime \prime}, \ell_{2 m(k)}^{\prime \prime}, \ell_{2 n(k)}^{\prime \prime}\right) \leq \\
& \omega\left[\bar{s}\left(\ell_{2 m(k)}^{\prime \prime}, \ell_{2 m(k)}^{\prime \prime}, \ell_{2 n(k)-1}^{\prime \prime}\right)+\bar{s}\left(\ell_{2 m(k)}^{\prime \prime}, \ell_{2 m(k)}^{\prime \prime}, \ell_{2 n(k)-1}^{\prime \prime}\right)+\bar{s}\left(\ell_{2 m(k)}^{\prime \prime}, \ell_{2 m(k)}^{\prime \prime}, \ell_{2 n(k)-1}^{\prime \prime}\right)\right]
\end{aligned}
$$

Letting $k \longrightarrow \infty$ in the above inequality, we have

Finally,

$$
\omega^{-1}(\varepsilon) \leq \limsup _{k \rightarrow \infty} \bar{s}\left(\ell_{2 m(k)+1}^{\prime \prime}, \ell_{2 m(k)+1}^{\prime \prime}, \ell_{2 n(k)}^{\prime \prime}\right) \text {. }
$$

$$
\begin{gathered}
\quad \bar{s}\left(\ell_{2 m(k)}^{\prime \prime}, \ell_{2 m(k)}^{\prime \prime}, \ell_{2 n(k)-1}^{\prime \prime}\right) \leq \\
\omega\left[\bar{s}\left(\ell_{2 m(k)+1}^{\prime \prime}, \ell_{2 m(k)+1}^{\prime \prime}, \ell_{2 m(k)}^{\prime \prime}\right)+\bar{s}\left(\ell_{2 m(k)+1}^{\prime \prime}, \ell_{2 m(k)+1}^{\prime \prime}, \ell_{2 m(k)}^{\prime \prime}\right)+\bar{s}\left(\ell_{2 n(k)-1}^{\prime \prime}, \ell_{2 n(k)-1}^{\prime \prime}, \ell_{2 m(k)}^{\prime \prime}\right)\right] . \\
\text { limsup } \bar{s}\left(\ell_{2 m(k)+1}^{\prime \prime}, \ell_{2 m(k)+1}^{\prime \prime}, \ell_{2 n(k)-1}^{\prime \prime}\right) \leq \omega(\varepsilon) . \quad \alpha\left(\Upsilon_{1} \ell_{2 n(k)-1}, \Upsilon_{1} \ell_{2 n(k)-1}, \Upsilon_{1} \ell_{2 n(k)-3}\right) \geq 1, \quad \Upsilon_{1} \ell_{2 n}(43) \quad \text { Also, }
\end{gathered}
$$

Letting $k \longrightarrow \infty$ in the above and using (39), we have

$$
\alpha\left(\Upsilon_{1} \ell_{2 n(k)-1}, \Upsilon_{1} \ell_{2 n(k)-1}, \Upsilon_{1} \ell_{2 n(k)-3}\right) \geq 1, \quad \Upsilon_{1} \ell_{2 n(k)-1} \perp \Upsilon_{1} \ell_{2 n(k)-3}
$$

We know that $2 n(k)-1 \geq 2 m(k)$ and

$$
\alpha\left(\Upsilon_{2} \ell_{2 n+2}, \Upsilon_{2} \ell_{2 n+2}, \Upsilon_{1} \ell_{2 n+1}\right)=\alpha\left(\Gamma_{2} \ell_{2 n+1}, \Gamma_{2} \ell_{2 n+1}, \Gamma_{1} \ell_{2 n}\right) \geq 1
$$

for all $n \in \mathbb{N}$. On the other hand, the pairs $\left(\Gamma_{1}, \Gamma_{2}\right)$ and $\left(\Gamma_{2}, \Gamma_{1}\right)$ are rectangular partially weakly $O$ - $\alpha$-admissible with respect to $\Upsilon_{1}$ and $\Upsilon_{2}$, respectively. So,

$\alpha\left(\Upsilon_{1} \ell_{2 n(k)-1}, \Upsilon_{1} \ell_{2 n(k)-1}, \Upsilon_{2} \ell_{2 n(k)-2}\right) \geq 1, \quad \Upsilon_{1} \ell_{2 n(k)-1} \perp \Upsilon_{2} \ell_{2 n(k)-2}$, $\alpha\left(\Upsilon_{2} \ell_{2 n(k)-2}, \Upsilon_{2} \ell_{2 n(k)-2}, \Upsilon_{1} \ell_{2 n(k)-3}\right) \geq 1, \quad \Upsilon_{2} \ell_{2 n(k)-2} \perp \Upsilon_{1} \ell_{2 n(k)-3}$,
Also,

$\alpha\left(\Upsilon_{1} \ell_{2 n(k)-1}, \Upsilon_{1} \ell_{2 n(k)-1}, \Upsilon_{1} \ell_{2 n(k)-3}\right) \geq 1, \quad \Upsilon_{1} \ell_{2 n(k)-1} \perp \Upsilon_{1} \ell_{2 n(k)-3}$, $\alpha\left(\Upsilon_{1} \ell_{2 n(k)-3}, \Upsilon_{1} \ell_{2 n(k)-3}, \Upsilon_{2} \ell_{2 n(k)-4}\right) \geq 1, \quad \Upsilon_{1} \ell_{2 n(k)-3} \perp \Upsilon_{2} \ell_{2 n(k)-4}$,

which imply that

$\alpha\left(\Upsilon_{1} \ell_{2 n(k)-1}, \Upsilon_{1} \ell_{2 n(k)-1}, \Upsilon_{2} \ell_{2 n(k)-4}\right) \geq 1, \Upsilon_{1} \ell_{2 n(k)-1} \perp \Upsilon_{2} \ell_{2 n(k)-4}$.

Continuing in this manner, we obtain that $\alpha\left(\Upsilon_{1} \ell_{2 n(k)-1}, \Upsilon_{1} \ell_{2 n(k)-1}, \Upsilon_{2} \ell_{2 m(k)}\right) \geq 1 \quad$ and $\Upsilon_{1} \ell_{2 n(k)-1} \perp$ $\Upsilon_{2} \ell_{2 m(k)}$. Now, we can apply (15) to acquire that

which imply that

$$
\begin{aligned}
& \pi\left(\omega\left(\bar{s}\left(\ell_{2 m(k)+1}^{\prime \prime}, \ell_{2 m(k)+1}^{\prime \prime}, \ell_{2 n(k)}^{\prime \prime}\right)\right)\right) \\
& \quad=\pi\left(\omega\left(\bar{s}\left(\Gamma_{1} \ell_{2 m(k)}, \Gamma_{1} \ell_{2 m(k)}, \Gamma_{2} \ell_{2 n(k)-1}\right)\right)\right) \\
& \quad \leq \pi\left(M\left(\ell_{2 m(k)}, \ell_{2 m(k)}, \ell_{2 n(k)-1}\right)\right)-\omega\left(M\left(\ell_{2 m(k)}, \ell_{2 m(k)}, \ell_{2 n(k)-1}\right)\right)
\end{aligned}
$$


10

Journal of Mathematics

where

$$
\begin{aligned}
& M\left(\ell_{2 m(k)}, \ell_{2 m(k)}, \ell_{2 n(k)-1}\right) \\
& \in\left\{\bar{s}\left(\Upsilon_{2} \ell_{2 m(k)}, \Upsilon_{2} \ell_{2 m(k)}, \Upsilon_{1} \ell_{2 n(k)-1}\right),\right. \\
& \omega^{-1}\left[\frac{\bar{s}\left(\Upsilon_{2} \ell_{2 m(k)}, \Upsilon_{2} \ell_{2 m(k)}, \Gamma_{1} \ell_{2 m(k)}\right)+\bar{s}\left(\Upsilon_{1} \ell_{2 n(k)-1}, \Upsilon_{1} \ell_{2 n(k)-1}, \Gamma_{2} \ell_{2 n(k)-1}\right)}{2}\right], \\
&\left.\omega^{-1}\left[\frac{\bar{s}\left(\Upsilon_{2} \ell_{2 m(k)}, \Upsilon_{2} \ell_{2 m(k)}, \Gamma_{2} \ell_{2 n(k)-1}\right)+\bar{s}\left(\Upsilon_{1} \ell_{2 n(k)-1}, \Upsilon_{1} \ell_{2 n(k)-1}, \Gamma_{1} \ell_{2 m(k)}\right)}{2}\right]\right\} \\
&=\left\{\bar{s}\left(\ell_{2 m(k)}^{\prime \prime}, \ell_{2 m(k)}^{\prime \prime}, \ell_{2 n(k)-1}^{\prime \prime}\right),\right. \\
& \omega^{-1}\left[\frac{\bar{s}\left(\ell_{2 m(k)}^{\prime \prime}, \ell_{2 m(k)}^{\prime \prime}, \ell_{2 m(k)+1}^{\prime \prime}\right)+\bar{s}\left(\ell_{2 n(k)-1}^{\prime \prime}, \ell_{2 n(k)-1}^{\prime \prime}, \ell_{2 n(k)}^{\prime \prime}\right)}{2}\right], \\
&\left.\omega^{-1}\left[\frac{\bar{s}\left(\ell_{2 m(k)}^{\prime \prime}, \ell_{2 m(k)}^{\prime \prime}, \ell_{2 n(k)}^{\prime \prime}\right)+\bar{s}\left(\ell_{2 n(k)-1}^{\prime \prime}, \ell_{2 n(k)-1}^{\prime \prime}, \ell_{2 m(k)-1}^{\prime \prime}\right)}{2}\right]\right\} .
\end{aligned}
$$

If

$$
M\left(\ell_{2 m(k)}, \ell_{2 m(k)}, \ell_{2 n(k)-1}\right)=\omega^{-1}\left[\frac{\bar{s}\left(\ell_{2 m(k)}^{\prime \prime}, \ell_{2 m(k)}^{\prime \prime}, \ell_{2 m(k)+1}^{\prime \prime}\right)+\bar{s}\left(\ell_{2 n(k)-1}^{\prime \prime}, \ell_{2 n(k)-1}^{\prime \prime}, \ell_{2 n(k)}^{\prime \prime}\right)}{2}\right]
$$

then from (35), we get that $\lim _{k \longrightarrow \infty} M\left(\ell_{2 m(k)}, \ell_{2 m(k)}, \quad \lim _{k \longrightarrow \infty} \bar{s}\left(\ell_{2 m(k)+1}^{\prime \prime}, \ell_{2 m(k)+1}^{\prime \prime}, \ell_{2 n(k)}^{\prime \prime}\right)=0\right.$, which contradicts $\left.\ell_{2 n(k)-1}\right)=0$. Hence, according to (49), we have (41).

$$
M\left(\ell_{2 m(k)}, \ell_{2 m(k)}, \ell_{2 n(k)-1}\right)=\omega^{-1}\left[\frac{\bar{s}\left(\ell_{2 m(k)}^{\prime \prime}, \ell_{2 m(k)}^{\prime \prime}, \ell_{2 n(k)}^{\prime \prime}\right)+\bar{s}\left(\ell_{2 n(k)-1}^{\prime \prime}, \ell_{2 n(k)-1}^{\prime \prime}, \ell_{2 m(k)+1}^{\prime \prime}\right)}{2}\right]
$$


then from (39) and (43), we get that

$$
\limsup _{k \rightarrow \infty} M\left(\ell_{2 m(k)}, \ell_{2 m(k)}, \ell_{2 n(k)-1}\right) \leq \omega^{-1}\left(\frac{\omega(\varepsilon)+\omega(\varepsilon)}{2}\right)=\varepsilon .
$$

Taking the limit as $k \longrightarrow \infty$ in (49), we have

$$
\begin{aligned}
& \pi(\varepsilon)=\pi\left(\omega\left(\omega^{-1}(\varepsilon)\right)\right) \\
& \leq \pi\left(\omega\left(\limsup _{k \longrightarrow \infty} \bar{s}\left(\ell_{2 m(k)+1}^{\prime \prime}, \ell_{2 m(k)+1}^{\prime \prime}, \ell_{2 n(k)}^{\prime \prime}\right)\right)\right) \\
& \leq \pi\left(\limsup _{k \longrightarrow \infty} \mathrm{M}\left(\ell_{2 m(k)}, \ell_{2 m(k)}, \ell_{2 n(k)-1}\right)\right)-\omega\left(\liminf _{k \rightarrow \infty} \mathrm{M}\left(\ell_{2 m(k)}, \ell_{2 m(k)}, \ell_{2 n(k)-1}\right)\right) \\
& \leq \pi(\varepsilon)-\omega\left(\liminf _{k \longrightarrow \infty} \mathrm{M}\left(\ell_{2 m(k)}, \ell_{2 m(k)}, \ell_{2 n(k)-1}\right)\right) \text {, }
\end{aligned}
$$

which implies that $\Phi\left(\liminf _{k \longrightarrow \infty} M\left(\ell_{2 m(k)}, \ell_{2 m(k)}\right.\right.$, $\left.\left.\ell_{2 n(k)-1}\right)\right) \leq 0$. Hence, $\liminf _{k \longrightarrow \infty} \bar{s}\left(\ell_{2 m(k)}, \ell_{2 m(k)}, \ell_{2 n(k)}\right)=0$, a contradiction to (39).

If

$$
M\left(\ell_{2 m(k)}, \ell_{2 m(k)}, \ell_{2 n(k)-1}\right)=\bar{s}\left(\ell_{2 m(k)}, \ell_{2 m(k)}, \ell_{2 n(k)-1}\right),
$$

then from (37), by taking the limit as $k \longrightarrow \infty$ in (49), we have

$$
\begin{aligned}
\pi(\varepsilon) & =\pi\left(\omega\left(\omega^{-1}(\varepsilon)\right)\right) \\
& \leq \pi\left(\omega\left(\limsup _{k \longrightarrow \infty} \bar{s}\left(\ell_{m(k)+1}^{\prime \prime}, \ell_{m(k)+1}^{\prime \prime}, \ell_{n(k)}^{\prime \prime}\right)\right)\right) \\
& \leq \pi\left(\limsup _{k \longrightarrow \infty} \bar{s}\left(\ell_{2 m(k)}^{\prime \prime}, \ell_{2 m(k)}^{\prime \prime}, \ell_{2 n(k)-1}^{\prime \prime}\right)\right)-\omega\left(\liminf _{k \rightarrow \infty} \bar{s}\left(\ell_{2 m(k)}^{\prime \prime}, \ell_{2 m(k)}^{\prime \prime}, \ell_{2 n(k)-1}^{\prime \prime}\right)\right) \\
& \leq \pi(\varepsilon)-\omega\left(\liminf _{k \longrightarrow \infty} \bar{s}\left(\ell_{2 m(k)}^{\prime \prime}, \ell_{2 m(k)}^{\prime \prime}, \ell_{2 n(k)-1}^{\prime \prime}\right)\right),
\end{aligned}
$$

which implies that $\Phi\left(\liminf _{k \longrightarrow \infty} \bar{s}\left(\ell_{2 m(k)}^{\prime \prime}, \ell_{2 m(k)}^{\prime \prime}\right.\right.$, $\left.\left.\ell_{2 n(k)-1}^{\prime \prime}\right)\right) \leq 0$. $\liminf _{k \longrightarrow \infty} \bar{s}\left(\ell_{2 m(k)}^{\prime \prime}, \ell_{2 m(k)}^{\prime \prime}, \ell_{2 n(k)}^{\prime \prime}\right)=0$. Therefore, from the rectangular inequality, we can conclude that $\liminf _{k \longrightarrow \infty} \bar{s}\left(\ell_{2 m(k)}^{\prime \prime}, \ell_{2 m(k)}^{\prime \prime}, \ell_{2 n(k)}^{\prime \prime}\right)=0$ which contradicts (39). Hence, $\left\{\ell_{n}^{\prime \prime}\right\}$ is a $\bar{s}$-Cauchy $O$-sequence.
Step 3. We will show that $\Gamma_{1}, \Gamma_{2}, \Upsilon_{1}$, and $\Upsilon_{2}$ have a point of coincidence.

Since $\left\{\ell_{n}^{\prime \prime}\right\}$ is a $\bar{s}$-Cauchy $O$-sequence in the $O$ - $\alpha$-complete $O-\bar{s}$-MS $\mathscr{L}$ and $\alpha\left(\ell_{k}^{\prime \prime}, \ell_{k}^{\prime \prime}, \ell_{k+1}^{\prime \prime}\right) \geq 1$, then there exists $\ell^{\prime \prime} \in \mathscr{L}$, such that

$$
\begin{aligned}
\lim _{n \longrightarrow \infty} \bar{s}\left(\ell_{2 n+1}^{\prime \prime}, \ell_{2 n+1}^{\prime \prime}, \ell^{\prime \prime}\right) & =\lim _{n \longrightarrow \infty} \bar{s}\left(\Upsilon_{1} \ell_{2 n+1}, \Upsilon_{1} \ell_{2 n+1}, \ell^{\prime \prime}\right)=\lim _{n \longrightarrow \infty} \bar{s}\left(\Gamma_{1} \ell_{2 n+1}, \Gamma_{1} \ell_{2 n+1}, \ell^{\prime \prime}\right)=0 \\
\lim _{n \longrightarrow \infty} \bar{s}\left(\ell_{2 n}^{\prime \prime}, \ell_{2 n}^{\prime \prime}, \ell^{\prime \prime}\right) & =\lim _{n \longrightarrow \infty} \bar{s}\left(\Upsilon_{2} \ell_{2 n}, \Upsilon_{2} \ell_{2 n}, \ell^{\prime \prime}\right)=\lim _{n \longrightarrow \infty} \bar{s}\left(\Gamma_{2} \ell_{2 n-1}, \Gamma_{2} \ell_{2 n-1}, \ell^{\prime \prime}\right)=0 .
\end{aligned}
$$


Hence,

$$
\Upsilon_{2} \ell_{2 n} \longrightarrow \ell^{\prime \prime} \text { and } \Gamma_{1} \ell_{2 n} \longrightarrow \ell^{\prime \prime} \text {, as } n \longrightarrow \infty \text {. }
$$

As $\left(\Gamma_{1}, \Upsilon_{2}\right)$ is $O$ - $\alpha$-compatible and $\alpha\left(\ell_{2 n}^{\prime \prime}, \ell_{2 n}^{\prime \prime}, \ell_{2 n+1}^{\prime \prime}\right) \geq 1$, so

$$
\lim _{n \rightarrow \infty} \bar{s}\left(\Upsilon_{2} \Gamma_{1} \ell_{2 n}, \Upsilon_{2} \Gamma_{1} \ell_{2 n}, \Gamma_{1} \Upsilon_{2} \ell_{2 n}\right)=0
$$

Moreover, from $\lim \bar{s}\left(\Gamma_{1} \ell_{2 n}, \Gamma_{1} \ell_{2 n}, \ell^{\prime \prime}\right)=0$, $\lim _{n \longrightarrow \infty} \bar{s}\left(\Upsilon_{2} \ell_{2 n}, \Upsilon_{2} \ell_{2 n}, \ell^{\prime \prime}\right)=0$, and the $O$ - $\alpha$-continuity of $\Upsilon_{2}$ and $\Gamma_{1}$, we acquire that

$$
\lim _{n \longrightarrow \infty} \bar{s}\left(\Upsilon_{2} \Gamma_{1} \ell_{2 n}, \Upsilon_{2} \Gamma_{1} \ell_{2 n}, \Upsilon_{2} \ell^{\prime \prime}\right)=0=\lim _{n \longrightarrow \infty} \bar{s}\left(\Gamma_{1} \Upsilon_{2} \ell_{2 n}, \Gamma_{1} \Upsilon_{2} \ell_{2 n}, \Gamma_{1} \ell^{\prime \prime}\right)
$$

By the rectangle inequality, we have

$$
\begin{aligned}
& \bar{s}\left(\Upsilon_{2} \ell^{\prime \prime}, \Upsilon_{2} \ell^{\prime \prime}, \Gamma_{1} \ell^{\prime \prime}\right) \\
\leq & \omega\left[\bar{s}\left(\Upsilon_{2} \ell^{\prime \prime}, \Upsilon_{2} \ell^{\prime \prime}, \Upsilon_{2} \Gamma_{1} \ell_{2 n}\right)+\bar{s}\left(\Upsilon_{2} \ell^{\prime \prime}, \Upsilon_{2} \ell^{\prime \prime}, \Upsilon_{2} \Gamma_{1} \ell_{2 n}\right)+\bar{s}\left(\Gamma_{1} \ell^{\prime \prime}, \Gamma_{1} \ell^{\prime \prime}, \Upsilon_{2} \Gamma_{1} \ell_{2 n}\right)\right] \\
\leq & \omega\left[\bar{s}\left(\Upsilon_{2} \ell^{\prime \prime}, \Upsilon_{2} \ell^{\prime \prime}, \Upsilon_{2} \Gamma_{1} \ell_{2 n}\right)+\bar{s}\left(\Upsilon_{2} \ell^{\prime \prime}, \Upsilon_{2} \ell^{\prime \prime}, \Upsilon_{2} \Gamma_{1} \ell_{2 n}\right)\right. \\
+ & \left.\omega \bar{s}\left(\Gamma_{1} \ell^{\prime \prime}, \Gamma_{1} \ell^{\prime \prime}, \Gamma_{1} \Upsilon_{2} \ell_{2 n}\right)+\bar{s}\left(\Gamma_{1} \ell^{\prime \prime}, \Gamma_{1} \ell^{\prime \prime}, \Gamma_{1} \Upsilon_{2} \ell_{2 n}\right)+\bar{s}\left(\Upsilon_{2} \Gamma_{1} \ell_{2 n}, \Upsilon_{2} \Gamma_{1} \ell_{2 n}, \Gamma_{1} \Upsilon_{2} \ell_{2 n}\right)\right] .
\end{aligned}
$$

Taking the limit as $n \longrightarrow \infty$ in (61), we deduce that

$$
\bar{s}\left(\Upsilon_{2} \ell^{\prime \prime}, \Upsilon_{2} \ell^{\prime \prime}, \Gamma_{1} \ell^{\prime \prime}\right) \leq 0 \text {, }
$$

which yields that $\Gamma_{1} \ell^{\prime \prime}=\Upsilon_{2} \ell^{\prime \prime}$, that is, $\ell^{\prime \prime}$ is a coincidence point of $\Gamma_{1}$ and $\Upsilon_{2}$.

Similarly, it can be proved that $\Gamma_{2} \ell^{\prime \prime}=\Upsilon_{1} \ell^{\prime \prime}$. Now, let $\alpha\left(\Upsilon_{1} \ell^{\prime \prime}, \Upsilon_{1} \ell^{\prime \prime}, \Upsilon_{2} \ell^{\prime \prime}\right) \geq 1$ and $\Upsilon_{1} \ell^{\prime \prime} \perp \Upsilon_{2} \ell^{\prime \prime}$. From (15), we have

$$
\pi\left(\omega\left(\bar{s}\left(\Gamma_{1} \ell^{\prime \prime}, \Gamma_{1} \ell^{\prime \prime}, \Gamma_{2} \ell^{\prime \prime}\right)\right)\right) \leq \pi\left(M\left(\ell^{\prime \prime}, \ell^{\prime \prime}, \ell^{\prime \prime}\right)\right)-\oplus\left(M\left(\ell^{\prime \prime}, \ell^{\prime \prime}, \ell^{\prime \prime}\right)\right),
$$

where

$$
\begin{aligned}
& M\left(\ell^{\prime \prime}, \ell^{\prime \prime}, \ell^{\prime \prime}\right) \in\left\{\bar{s}\left(\Upsilon_{2} \ell^{\prime \prime}, \Upsilon_{2} \ell^{\prime \prime}, \Upsilon_{1} \ell^{\prime \prime}\right),\right. \omega^{-1}\left[\frac{\bar{s}\left(\Upsilon_{2} \ell^{\prime \prime}, \Upsilon_{2} \ell^{\prime \prime}, \Upsilon_{1} \ell^{\prime \prime}\right)+\bar{s}\left(\Upsilon_{1} \ell^{\prime \prime}, \Upsilon_{1} \ell^{\prime \prime}, \Gamma_{2} \ell^{\prime \prime}\right)}{2}\right], \\
&\left.\omega^{-1}\left[\frac{\bar{s}\left(\Upsilon_{2} \ell^{\prime \prime}, \Upsilon_{2} \ell^{\prime \prime}, \Gamma_{2} \ell^{\prime \prime}\right)+\bar{s}\left(\Upsilon_{1} \ell^{\prime \prime}, \Upsilon_{1} \ell^{\prime \prime}, \Gamma_{1} \ell^{\prime \prime}\right)}{2}\right]\right\} \\
&=\left\{\bar{s}\left(\Gamma_{1} \ell^{\prime \prime}, \Gamma_{1} \ell^{\prime \prime}, \Gamma_{2} \ell^{\prime \prime}\right), 0, \omega^{-1}\left[\bar{s}\left(\Gamma_{1} \ell^{\prime \prime}, \Gamma_{1} \ell^{\prime \prime}, \Gamma_{2} \ell^{\prime \prime}\right)\right]\right\} .
\end{aligned}
$$

In all three cases, the above inequality yields that $\Gamma_{1} \ell^{\prime \prime}=\Gamma_{2} \ell^{\prime \prime}=\Upsilon_{2} \ell^{\prime \prime}=\Upsilon_{1} \ell^{\prime \prime}$.

In what follows, we replace the obligation of $O$ - $\alpha$-continuity of $\Gamma_{1}, \Gamma_{2}, \Upsilon_{1}$, and $\Upsilon_{2}$ by an hypothesis on the underlying space and replace the $O$ - $\alpha$-compatibility by weak compatibility.

Theorem 2. Let $(\mathscr{L}, \bar{s}, \omega, \perp)$ be an O- $\alpha$-regular O- $\alpha$-complete $\bar{s}$-MS, $\alpha: \mathscr{L} \times \mathscr{L} \times \mathscr{L} \longrightarrow[0, \infty)$, and let $\Gamma_{1}, \Gamma_{2}, \Upsilon_{1}, \Upsilon_{2}: \mathscr{L} \longrightarrow \mathscr{L}$ be such that $\Gamma_{1}(\mathscr{L}) \subseteq \Upsilon_{1}(\mathscr{L})$,
$\Gamma_{2}(\mathscr{L}) \subseteq \Upsilon_{2}(\mathscr{L})$, and $\Upsilon_{1}(\mathscr{L})$ and $\Upsilon_{2}(\mathscr{L})$ are two $\bar{s}$-closed subsets of $\mathscr{L}$. Suppose that

$$
\pi\left(\omega^{2}\left(\bar{s}\left(\Gamma_{1} \ell, \Gamma_{1} \ell, \Gamma_{2} \ell^{\prime}\right)\right)\right) \leq \pi\left(M\left(\ell, \ell, \ell^{\prime}\right)\right)-\oplus\left(M\left(\ell, \ell, \ell^{\prime}\right)\right),
$$

for all $\ell$ and $\ell^{\prime}$ with $\alpha\left(\Upsilon_{2} \ell, \Upsilon_{2} \ell, \Upsilon_{1} \ell^{\prime}\right) \geq 1$ and $\Upsilon_{2} \ell \perp \Upsilon_{1} \ell^{\prime}$. Then, the pairs $\left(\Gamma_{1}, \Gamma_{2}\right)$ and $\left(\Gamma_{2}, \Upsilon_{1}\right)$ admit a coincidence point $\ell^{\prime \prime}$ in $\mathscr{L}$ whenever the pairs $\left(\Gamma_{1}, \Upsilon_{2}\right)$ and $\left(\Gamma_{2}, \Upsilon_{1}\right)$ are weakly compatible and the pairs $\left(\Gamma_{1}, \Gamma_{2}\right)$ and $\left(\Gamma_{2}, \Upsilon_{1}\right)$ are rectangular 
partially weakly $O-\alpha-\Upsilon_{1}$-admissible and $O-\alpha-\Upsilon_{2}$-admissible, respectively. Moreover, if $\alpha\left(\Upsilon_{2} \ell^{\prime \prime}, \Upsilon_{2} \ell^{\prime \prime}, \Upsilon_{1} \ell^{\prime \prime}\right) \geq 1$ and $\Upsilon_{2} \ell^{\prime \prime} \perp \Upsilon_{1} \ell^{\prime \prime}$, then $\ell^{\prime \prime} \in \mathscr{L}$ is a coincidence point of $\Gamma_{1}, \Gamma_{2}, \Upsilon_{1}$, and $\Upsilon_{2}$.

Proof. From the proof of Theorem (15), for some $\ell^{\prime \prime} \in \mathscr{L}$, we have

$$
\lim _{k \rightarrow \infty} \bar{s}\left(\ell_{k}^{\prime \prime}, \ell_{k}^{\prime \prime}, \ell^{\prime \prime}\right)=0 .
$$

Since $\Upsilon_{1}(\mathscr{L})$ is $\bar{s}$-closed and $\left\{\ell_{2 n+1}{ }^{\prime \prime}\right\} \subseteq \Upsilon_{1}(\mathscr{L})$, therefore, $\ell^{\prime \prime} \in \Upsilon_{1}(\mathscr{L})$. Hence, for some $u \in \mathscr{L}$, one has $\ell^{\prime \prime}=\Upsilon_{1} u$, and $\lim _{n \longrightarrow \infty} \bar{s}\left(\ell_{2 n+1}^{\prime \prime}, \ell_{2 n+1}^{\prime \prime}, \Upsilon_{1} u\right)=\lim _{n \longrightarrow \infty} \bar{s}\left(\Upsilon_{1} \ell_{2 n+1}, \Upsilon_{1} \ell_{2 n+1}, \Upsilon_{1} u\right)=0$

Similarly, for $v \in \mathscr{L}$, we have $\ell^{\prime \prime}=\Upsilon_{1} u=\Upsilon_{2} v$ and

$\lim _{n \longrightarrow \infty} \bar{s}\left(\ell_{2 n}^{\prime \prime}, \ell_{2 n}^{\prime \prime}, \Upsilon_{2} v\right)=\lim _{n \longrightarrow \infty} \bar{s}\left(\Upsilon_{2} \ell_{2 n}, \Upsilon_{2} \ell_{2 n}, \Upsilon_{2} v\right)=0$

We shall show that $v$ is a coincidence point of $\Gamma_{1}$ and $\Upsilon_{2}$.

Since $\Upsilon_{1} \ell_{2 n+1} \longrightarrow \ell^{\prime \prime}=\Upsilon_{2} v$, as $n \longrightarrow \infty$, from $O$ - $\alpha$-regularity of $\mathscr{L}, \alpha\left(\Upsilon_{1} \ell_{2 n+1}, \Upsilon_{1} \ell_{2 n+1}, \Upsilon_{2} v\right) \geq 1$. Therefore, from (65), we have

$$
\pi\left(\omega^{2}\left(\bar{s}\left(\Gamma_{1} v, \Gamma_{1} v, \Gamma_{2} \ell_{2 n+1}\right)\right)\right) \leq \pi\left(M\left(v, v, \ell_{2 n+1}\right)\right)-\oplus\left(M\left(v, v, \ell_{2 n+1}\right)\right)
$$

where

$$
\begin{aligned}
& M\left(v, v, \ell_{2 n+1}\right) \in\left\{\bar{s}\left(\Upsilon_{2} v, \Upsilon_{2} v, \Upsilon_{1} \ell_{2 n+1}\right), \omega^{-1}\left(\frac{\bar{s}\left(\Upsilon_{2} v, \Upsilon_{2} v, \Gamma_{1} v\right)+\bar{s}\left(\Upsilon_{1} \ell_{2 n+1}, \Upsilon_{1} \ell_{2 n+1}, \Gamma_{2} \ell_{2 n+1}\right)}{2}\right)\right], \\
& \left.\omega^{-1}\left(\frac{\bar{s}\left(\Upsilon_{2} v, \Upsilon_{2} v, \Gamma_{2} \ell_{2 n+1}\right)+\bar{s}\left(\Upsilon_{1} \ell_{2 n+1}, \Upsilon_{1} \ell_{2 n+1}, \Gamma_{1} v\right)}{2}\right]\right\} \\
& \quad=\left\{\bar{s}\left(\ell^{\prime \prime}, \ell^{\prime \prime}, \ell_{2 n+1}^{\prime \prime}\right), \omega^{-1}\left(\frac{\bar{s}\left(\ell^{\prime \prime}, \ell^{\prime \prime}, \Gamma_{1} v\right)+\bar{s}\left(\ell_{2 n+1}^{\prime \prime}, \ell_{2 n+1}^{\prime \prime}, \ell_{2 n}^{\prime \prime}\right)}{2}\right],\right. \\
& \left.\omega^{-1}\left(\frac{\bar{s}\left(\ell^{\prime \prime}, \ell^{\prime \prime}, \ell_{2 n}^{\prime \prime}\right)+\bar{s}\left(\ell_{2 n+1}^{\prime \prime}, \ell_{2 n+1}^{\prime \prime}, \Gamma_{1} v\right)}{2}\right]\right\} .
\end{aligned}
$$

From Lemma 1,

$$
\omega^{-1}\left[\frac{\omega^{-2}\left[\bar{s}\left(\ell^{\prime \prime}, \ell^{\prime \prime}, \Gamma_{1} v\right)\right]}{2}\right] \leq \liminf _{n \longrightarrow \infty} M\left(v, v, \ell_{2 n+1}\right) \leq \limsup _{n \longrightarrow \infty} M\left(v, v, \ell_{2 n+1}\right) \leq \omega\left[\bar{s}\left(\ell^{\prime \prime}, \ell^{\prime \prime}, \Gamma_{1} v\right)\right]
$$

Taking the upper limit as $n \longrightarrow \infty$ in (69), using Lemma 1 and the continuity of $\pi$ and $\omega$, we acquire that

$$
\begin{aligned}
\pi\left(\omega^{2}\left[\bar{s}\left(\Gamma_{1} v, \Gamma_{1} v, \ell^{\prime \prime}\right)\right]\right) & \leq \pi\left(\bar{s}\left(\Gamma_{1} v, \Gamma_{1} v, \Gamma_{2} \ell_{2 n+1}\right)\right) \\
& \leq \pi\left(\omega^{-1}\left(\frac{\omega^{2}\left[\bar{s}\left(\ell^{\prime \prime}, \ell^{\prime \prime}, \Gamma_{1} v\right)\right]}{2}\right)\right)-\omega\left(\liminf _{n \longrightarrow \infty} M\left(v, v, \ell_{2 n+1}\right)\right) \\
& \leq \pi\left(\omega\left(\bar{s}\left(\ell^{\prime \prime}, \ell^{\prime \prime}, \Gamma_{1} v\right)\right)\right)-\omega\left(\liminf _{n \longrightarrow \infty} M\left(v, v, \ell_{2 n+1}\right)\right) \\
& \leq \pi\left(\omega^{2}\left(\bar{s}\left(\Gamma_{1} v, \Gamma_{1} v, \ell^{\prime \prime}\right)\right)\right)-\omega\left(\liminf _{n \longrightarrow \infty} M\left(v, v, \ell_{2 n+1}\right)\right),
\end{aligned}
$$


which implies that $\omega^{-1}\left[\omega^{-2}\left[\bar{s}\left(\ell^{\prime \prime}, \ell^{\prime \prime}, \Gamma_{1} v\right)\right] / 2\right]=0$. Hence, $\Gamma_{1} v=\ell^{\prime \prime}=\Upsilon_{2} v$.

As $\Gamma_{1}$ and $\Upsilon_{2}$ are weakly compatible, we have $\Gamma_{1} \ell^{\prime \prime}=\Gamma_{1} \Upsilon_{2} v=\Upsilon_{2} \Gamma_{1} v=\Upsilon_{2} \ell^{\prime \prime}$. Thus, $\ell^{\prime \prime}$ is a coincidence point of $\Gamma_{1}$ and $\Upsilon_{2}$.

Analogously, it can be shown that $\ell^{\prime \prime}$ is a coincidence point of the pair $\left(\Gamma_{2}, \Upsilon_{1}\right)$.

Example 5. Let $\mathscr{L}=[0,84]$, and $\bar{s}$ on $\mathscr{L}$ be given by $\bar{s}\left(\ell, \ell^{\prime}, \ell^{\prime \prime}\right)=e^{\left|\ell-\ell^{\prime \prime}\right|+\left|\ell^{\prime}-\ell^{\prime \prime}\right|}-1$, for all $\ell, \ell^{\prime}, \ell^{\prime \prime} \in \mathscr{L}$, with $\omega(t)=e^{t}-1$. Let $\alpha: \mathscr{L} \times \mathscr{L} \times \mathscr{L} \longrightarrow[0, \infty)$ be given by $\alpha\left(\ell, \ell^{\prime}, \ell^{\prime \prime}\right)=e^{\ell+\ell^{\prime}-\ell^{\prime \prime}}$ and $\ell \perp \ell^{\prime}$, iff $\ell \leq \ell^{\prime}$. Define self-maps $\Gamma_{1}, \Gamma_{2}, \Upsilon_{1}$, and $\Upsilon_{2}$ on $\mathscr{L}$ by

$$
\begin{aligned}
& \Gamma_{1} \ell=\ln \left(1+\left(\frac{\ell}{100}\right)^{2}\right), \quad \Upsilon_{1} \ell=e^{\ell}-1, \\
& \Gamma_{2} \ell=\ln \left(1+\left(\frac{\ell}{4}\right)^{2}\right), \quad \Upsilon_{2} \ell=e^{25 \ell}-1 .
\end{aligned}
$$

Let $\ell, \ell^{\prime} \in \mathscr{L}$ be such that $\ell^{\prime} \in \Upsilon_{1}^{-1} \Gamma_{1} \ell$, that is, $\Upsilon_{1} \ell^{\prime}=\Gamma_{1} \ell$. By the definition of $\Gamma_{1}$ and $\Upsilon_{1}$, we have $e^{\ell^{\prime}}-1=\ln$ $\left(1+(\ell / 100)^{2}\right)$, and so, $\ell^{\prime}=\ln \left(1+\ln \left(1+(\ell / 100)^{2}\right)\right)$. Therefore,

$$
\Gamma_{1} \ell=\ln \left(1+\left(\frac{\ell}{100}\right)^{2}\right) \geq \ln \left(1+\left(\frac{\ln \left(1+\ln \left(1+(\ell / 100)^{2}\right)\right)}{4}\right)^{2}\right)=\ln \left(1+\left(\frac{\ell^{\prime}}{4}\right)^{2}\right)=\Gamma_{2} \ell^{\prime}
$$

Thus, $\alpha\left(\Gamma_{1} \ell, \Gamma_{1} \ell, \Gamma_{2} \ell^{\prime}\right) \geq 1$ and $\Gamma_{2} \ell^{\prime} \perp \Gamma_{1} \ell$. Hence, $\left(\Gamma_{1}, \Gamma_{2}\right)$ is partially weakly $O-\alpha-\Upsilon_{1}$-admissible.

To prove that $\left(\Gamma_{2}, \Gamma_{1}\right)$ is partially weakly $O-\alpha-\Upsilon_{2}$-admissible, let $\ell, \ell^{\prime} \in \mathscr{L}$ be such that $\ell^{\prime} \in \Upsilon_{2}^{-1} \Gamma_{2} \ell$, that is, $\Upsilon_{2} \ell^{\prime}=\Gamma_{2} \ell$. Hence, we have $e^{25 \ell^{\prime}}-1=\ln \left(1+(\ell / 4)^{2}\right)$, and so, $\ell^{\prime}=\ln \left(1+\ln \left(1+(\ell / 4)^{2}\right)\right) / 25$. Therefore,

$$
\Gamma_{2} \ell=\ln \left(1+\left(\frac{\ell}{4}\right)^{2}\right) \geq \ln \left(1+\left(\frac{\ln \left(1+\ln \left(1+(\ell / 4)^{2}\right)\right) / 25}{100}\right)^{2}\right)=\ln \left(1+\left(\frac{\ell^{\prime}}{100}\right)^{2}\right)=\Gamma_{1} \ell^{\prime}
$$

Thus, $\alpha\left(\Gamma_{2} \ell, \Gamma_{2} \ell, \Gamma_{1} \ell^{\prime}\right) \geq 1$ and $\Gamma_{1} \ell^{\prime} \perp \Gamma_{2} \ell$. Furthermore,

$$
\Gamma_{1} \mathscr{L}=\Upsilon_{1} \mathscr{L}=\Gamma_{2} \mathscr{L}=\Upsilon_{2} \mathscr{L} .
$$

Define $\pi, \emptyset:[0, \infty) \longrightarrow[0, \infty)$ as $\pi(t)=\ln (1+\ln (1+$ $t)$ ) and $₫(t)=1 / 100 t$ for all $t \in[0, \infty)$.

Using the mean value theorem, for all $\ell$ and $\ell^{\prime}$ with $\alpha\left(\Upsilon_{2} \ell, \Upsilon_{2} \ell, \Upsilon_{1} \ell^{\prime}\right) \geq 1$, we have

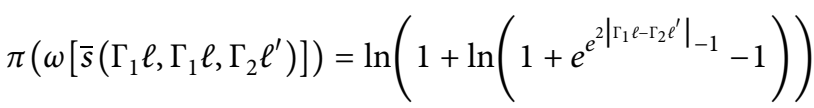

$$
\begin{aligned}
& =2\left|\ln \left(1+\left(\frac{\ell}{100}\right)^{2}\right)-\ln \left(1+\left(\frac{\ell^{\prime}}{4}\right)^{2}\right)\right| \leq 2\left|\frac{\ell}{100}-\frac{\ell^{\prime}}{4}\right| \\
& \leq 2 \frac{\left|\ell-25 \ell^{\prime}\right|}{100} \leq 2 \frac{1}{100}\left|e^{\ell}-1-\left(e^{25 \ell^{\prime}}-1\right)\right| \\
& \leq 2 \frac{1}{100}\left|\Upsilon_{2} \ell-\Upsilon_{1} \ell^{\prime}\right| \leq \frac{1}{100}\left(e^{2\left|\Upsilon_{2} \ell-\Upsilon_{1} e^{\prime}\right|}-1\right) \\
& \leq \ln \left(1+\ln \left(1+\left(e^{2\left|\Upsilon_{2} \ell-\Upsilon_{1} \ell^{\prime}\right|}-1\right)\right)\right)-\frac{1}{100}\left(e^{2\left|\Upsilon_{2} \ell-\Upsilon_{1} \ell^{\prime}\right|}-1\right) \\
& =\pi\left(\bar{s}\left(\Upsilon_{2} \ell, \Upsilon_{2} \ell, \Upsilon_{1} \ell^{\prime}\right)\right)-\Phi\left(\bar{s}\left(\Upsilon_{2} \ell, \Upsilon_{2} \ell, \Upsilon_{1} \ell^{\prime}\right)\right) \text {. }
\end{aligned}
$$


Thus, (15) is true for all $\ell, \ell^{\prime} \in \mathscr{L}$ and $M\left(\ell, \ell, \ell^{\prime}\right)=\bar{s}\left(\Upsilon_{2} \ell, \Upsilon_{2} \ell, \Upsilon_{1} \ell^{\prime}\right)$. Therefore, all the conditions of Theorem 1 are satisfied. Moreover, 0 is a coincidence point of $\Gamma_{1}, \Gamma_{2}, \Upsilon_{1}$, and $\Upsilon_{2}$.
We arrange the following example, which supports Theorem 2, based on Example 2.8 of [22].

Example 6. Consider the set $X=\{1,2,3,4\}$ and choose

$$
\begin{aligned}
\Gamma_{1} & =\left(\begin{array}{llll}
1 & 2 & 3 & 4 \\
2 & 1 & 3 & 3
\end{array}\right), \\
\Gamma_{2} & =\left(\begin{array}{llll}
1 & 2 & 3 & 4 \\
2 & 3 & 1 & 3
\end{array}\right), \\
\Upsilon_{1} & =\left(\begin{array}{llll}
1 & 2 & 3 & 4 \\
2 & 3 & 1 & 4
\end{array}\right), \\
\Upsilon_{2} & =\left(\begin{array}{llll}
1 & 2 & 3 & 4 \\
2 & 1 & 3 & 4
\end{array}\right), \\
d(x, y) & =\left\{\begin{array}{lll}
0, & & x=y \\
x+y, & \text { otherwise, }
\end{array}\right.
\end{aligned}
$$

Define the mapping $\alpha: X \times X \times X \longrightarrow \mathbb{R}$ and the relation $\perp$ on $X \times X$ by

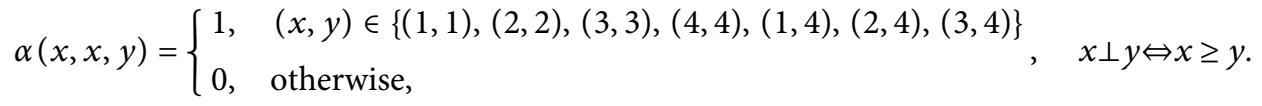

Let $\pi, \omega:[0, \infty) \longrightarrow[0, \infty)$ be defined by $\pi(t)=\sqrt{t} e^{t}$ and $\omega(t)=\tanh t$.

Obviously,

(1) $(\mathscr{L}, \bar{s}, \omega(t)=t, \perp)$ is an $O$ - $\alpha$-regular $O$ - $\alpha$-complete $\bar{s}$-MS. Indeed, let $\ell_{n} \longrightarrow \ell, \quad \ell_{n} \perp \ell_{n+1}$ and $\alpha\left(\ell_{n}, \ell_{n}, \ell_{n+1}\right) \geq 1$ for all $n \in \mathbb{N}$. Then, $\ell_{n}=\ell_{n+1}$ for all $n \in \mathbb{N}$. So, $(\mathscr{L}, \bar{s}, \omega(t)=t, \perp)$ is $O$ - $\alpha$-regular.

(2) $\Gamma_{1}(\mathscr{L}) \subseteq \Upsilon_{1}(\mathscr{L}), \quad \Gamma_{2}(\mathscr{L}) \subseteq \Upsilon_{2}(\mathscr{L})$, and $\Upsilon_{1}(\mathscr{L})$ and $\Upsilon_{2}(\mathscr{L})$ are two $\bar{s}$-closed subsets of $\mathscr{L}$

(3)

$$
\pi\left(\omega^{2}\left(\bar{s}\left(\Gamma_{1} \ell, \Gamma_{1} \ell, \Gamma_{2} \ell^{\prime}\right)\right)\right) \leq \pi\left(M\left(\ell, \ell, \ell^{\prime}\right)\right)-\varpi\left(M\left(\ell, \ell, \ell^{\prime}\right)\right),
$$

for all $\ell$ and $\ell^{\prime}$ with $\alpha\left(\Upsilon_{2} \ell, \Upsilon_{2} \ell, \Upsilon_{1} \ell^{\prime}\right) \geq 1$ and $\Upsilon_{2} \ell \perp \Upsilon_{1} \ell^{\prime}$, i.e., for all $\ell$ and $\ell^{\prime}$ with $\Upsilon_{2} \ell=\Upsilon_{1} \ell^{\prime}$.

(4) The pairs $\left(\Gamma_{2}, \Gamma_{1}\right)$ and $\left(\Gamma_{2}, \Gamma_{1}\right)$ are weakly compatible

(5) The pair $\left(\Gamma_{2}, \Gamma_{1}\right)$ is rectangular partially weakly $O-\alpha-\Upsilon_{1}$-admissible

(6) The pair $\left(\Gamma_{2}, \Gamma_{1}\right)$ is rectangular partially weakly $O-\alpha-\Upsilon_{2}$-admissible

Note that in parts 5 and 6 , it is sufficient to take $\ell=1$.

Therefore, $\Gamma_{1}, \Gamma_{2}, \Upsilon_{1}$, and $\Upsilon_{2}$ satisfy all the conditions given in Theorem 2 . Thus, 1, 2, and 3 are coincidence points of the pairs $\left(\Gamma_{1}, \Upsilon_{2}\right)$ and $\left(\Gamma_{2}, \Upsilon_{1}\right)$. Moreover, $\alpha\left(\Upsilon_{2} 1, \Upsilon_{2} 1, \Upsilon_{1} 1\right) \geq 1$ and $\Upsilon_{2} 1 \perp \Upsilon_{1} 1$, so $1 \in \mathscr{L}$ is a coincidence point of $\Gamma_{1}, \Gamma_{2}, \Upsilon_{1}$, and $\Upsilon_{2}$.

\section{Application}

In this part of the research, we study the following boundary value problem:

$$
\begin{cases}\nu^{\prime \prime}(\mu)=\mathscr{G}_{1}(\mu, \nu(\mu)), & \mu \in[0,1], \\ \nu^{\prime \prime}(\mu)=\mathscr{G}_{2}(\mu, \nu(\mu)), & \mu \in[0,1], \\ \nu(0)=v(1)=0, & \end{cases}
$$

where $\mathscr{G}_{1}, \mathscr{G}_{2}:[0,1] \times \mathbb{R} \longrightarrow \mathbb{R}$ are the continuous functions.

The above system is metamorphosed to the following system of Fredholm integral equations: 


$$
\left\{\begin{array}{l}
\nu(\mu)=-\int_{0}^{1} K(\mu, t) \mathscr{G}_{1}(t, v(t)) \mathrm{d} t \\
\nu(\mu)=-\int_{0}^{1} K(\mu, t) \mathscr{G}_{2}(t, v(t)) \mathrm{d} t
\end{array}\right.
$$

where the kernel is given by

$$
K(\mu, t)= \begin{cases}t(1-\mu), & \text { if } t \in[0, \mu], \\ \mu(1-t), & \text { if } t \in[\mu, 1] .\end{cases}
$$

See [23] for details.

Now, we present an existence result for a common solution of $(82)$ in $Q=C(I, \mathbb{R})$ (the set of continuous real functions defined on $I=[0,1])$. Note that we consider the space $\mathbb{Q}$, with the $\bar{s}$-metric given by

$$
\bar{s}(\zeta, \eta, \theta)=\max _{t \in I}(|\zeta(t)-\theta(t)|+|\eta(t)-\theta(t)|)^{p},
$$

for all $\zeta, \eta, \theta \in \mathbb{Q}$ which is $O-\alpha$-complete $O-\bar{s}$-MS. Note that $\omega(t)=2^{p-1} t, \alpha\left(\ell, \ell, \ell^{\prime}\right)=1$, and $\ell \perp \ell^{\prime}$ iff $\ell \leq \ell^{\prime}$.

Define $\Gamma_{1}, \Gamma_{2}: \mathbb{Q} \longrightarrow \mathbb{Q}$ by

$$
\left\{\begin{array}{l}
\Gamma_{1} \nu(\mu)=-\int_{0}^{1} K(\mu, t) \mathscr{G}_{1}(t, v(t)) \mathrm{d} t \\
\Gamma_{2} \nu(\mu)=-\int_{0}^{1} K(\mu, t) \mathscr{G}_{2}(t, v(t)) \mathrm{d} t
\end{array}\right.
$$

for all $\nu \in \mathbb{Q}$ and $\mu \in I$.

Clearly, a function $u \in \mathbb{Q}$ is a common solution of (82) if and only if it is a common fixed point of $\Gamma_{1}, \Gamma_{2}$.

Consider the following obligations:

(C1) For all $u, v \in \mathbb{Q}$ and for all $t \in I$,

$$
\left(\left|\mathscr{G}_{1}(t, u)-\mathscr{G}_{2}(t, v)\right|\right) \leq \sqrt[p]{\frac{\beta}{2^{2 p-2}}(|u(t)-v(t)|)} .
$$

$(\mathrm{C} 2) \mathscr{G}_{1}(t,),. \mathscr{G}_{2}(t,):. \mathbb{R} \longrightarrow \mathbb{R}$ are continuous for all $t \in[0,1]$

Theorem 3. Assume that the above presumptions (C1) and (C2) hold. Then, the system (82) possesses a common solution in Q.

Proof. To show that all assumptions of Theorem 2 are satisfied, it remains to prove that $\Gamma_{1}, \Gamma_{2}$ satisfy the contractive condition in Theorem 2. Let $v, \varrho \in \mathbb{Q}$. For each $\mu \in I$, we have

$$
\begin{aligned}
& \left(\left|\Gamma_{1} \nu(\mu)-\Gamma_{2} \varrho(\mu)\right|\right)^{p} \\
= & \left(\left|\int_{0}^{1} K(\mu, t) \mathscr{G}_{1}(t, v(t)) \mathrm{d} t-\int_{0}^{1} K(\mu, t) \mathscr{G}_{2}(t, \varrho(t)) \mathrm{d} t\right|\right)^{p} \\
\leq & \left(\left(\int_{0}^{1} \mid\left(\left.K(\mu, t)\right|^{q} \mathrm{~d} t\right)^{1 / q}\left(\int_{0}^{1}\left(\left|\mathscr{G}_{1}(t, \nu(t))-\mathscr{G}_{2}(t, \varrho(t))\right|\right)^{p} \mathrm{~d} t\right)^{1 / p}\right)^{p}\right. \\
\leq & \left(\int_{0}^{1} \mid\left(\left.K(\mu, t)\right|^{q} \mathrm{~d} t\right)^{p / q} \int_{0}^{1}\left(\left|\mathscr{G}_{1}(t, v(t))-\mathscr{G}_{2}(t, \varrho(t))\right|\right)^{p} \mathrm{~d} t\right. \\
\leq & \left(\int_{0}^{1} \mid\left(\left.K(\mu, t)\right|^{q} \mathrm{~d} t\right)^{p / q} \int_{0}^{1} \frac{\beta}{2^{2 p-2}}(|v(t)-\varrho(t)|)^{p} \mathrm{~d} t\right. \\
\leq & \left(\int_{0}^{1} \mid\left(\left.K(\mu, t)\right|^{q} \mathrm{~d} t\right)^{p / q} \int_{0}^{1} \frac{\beta}{2^{2 p-2}} \bar{s}(\nu, v, \varrho) \mathrm{d} t .\right.
\end{aligned}
$$


Via a careful calculation, we get that

$$
\int_{0}^{1}|K(\mu, t)|^{q} \mathrm{~d} t=\frac{(1-\mu)^{q} \mu^{q+1}+\mu^{q}(1-\mu)^{q+1}}{q+1}, \quad \mu \in[0,1] .
$$

So, we acquire that

$$
\left(\left|\Gamma_{1} \nu(\mu)-\Gamma_{2} \varrho(\mu)\right|\right)^{p} \leq \frac{\beta}{2^{2 p-2}} \frac{(1-\mu)^{q} \mu^{q+1}+\mu^{q}(1-\mu)^{q+1}}{q+1} \bar{s}(\nu, \nu, \varrho) .
$$

Taking the supremum on $\mu \in[0,1]$, we deduce that

$$
\bar{s}\left(\Gamma_{1} \nu, \Gamma_{1} \nu, \Gamma_{2} \varrho\right) \leq \frac{\beta}{2^{2 p-2}} \frac{\bar{s}(\nu, \nu, \rho)}{(q+1)} .
$$

Now, by taking $\pi(t)=t, \quad \omega(t)=2^{p-1} t, \quad$ and $\omega(t)=\left(1-\beta / 2^{2 p-2}\right) t$, we infer that

$$
\pi\left(\omega^{2}\left(\bar{s}\left(\Gamma_{1} \nu, \Gamma_{1} \nu, \Gamma_{2} \varrho\right)\right)\right) \leq \pi(\bar{s}(\nu, \nu, \varrho))-\Phi(\bar{s}(\nu, \nu, \varrho)) .
$$

Thus, all the hypotheses of Theorem 2 are fulfilled, and we deduce the existence of an element $u \in Q$, such that $u=\Gamma_{1} u=\Gamma_{2} u$. Note that regularity of the space $Q$ can be done as in [24].

\section{Conclusions}

In this study, we prove certain coincidence point theorems in an orthogonal extended $S$-metric space for four weakly $O$ - $\alpha$-admissible contractive mappings. Many authors consider spaces with an extrastructure, such as partially ordered spaces, graphical spaces, and orthogonal spaces, in addition to working on different spaces and different contractive conditions to avoid exploring the contractive conditions with all elements in the space. In reality, they significantly reduce the calculations in contractive conditions. Only elements that are orthogonal to each other are included in this study. Also, $\alpha$-admissibility is a generalization of monotonicity in the regular case.

\section{Data Availability}

No data were used to support this study.

\section{Conflicts of Interest}

The authors declare that there are no conflicts of interest.

\section{Authors' Contributions}

All authors contributed equally to the writing of this study. All authors read and approved the final manuscript.

\section{References}

[1] S. Banach, "Sur les opérations dans les ensembles abstraits et leur application aux équations intégrales," Fundamenta Mathematicae, vol. 3, pp. 133-181, 1922.
[2] M. Aslantas, H. Sahin, and I. Altun, "Best proximity point theorems for cyclic p-contractions with some consequences and applications," Nonlinear Analysis Modelling and Control, vol. 26, no. 1, pp. 113-129, 2021.

[3] A. Dehici and N. Redjel, "Some fixed point results for nonexpansive mappings in Banach spaces," Journal of Nonlinear Functional Analysis, vol. 2020, Article ID 36, 2020.

[4] B. C. Dhage, "Generalized metric space and mapping with fixed point," Bulletin of the Calcutta Mathematical Society, vol. 84, pp. 329-336, 1992.

[5] Z. Mustafa and B. Sims, "A new approach to generalized metric spaces," Journal of Nonlinear and Convex Analysis, vol. 7, pp. 289-297, 2006.

[6] S. G. Matthews, "Partial metric topology," Annals of the New York Academy of Sciences, vol. 728, no. 1, pp. 183-197, 1994.

[7] L. G. Huang and X. Zhang, "Cone metric spaces and fixed point theorems of contractive mappings," Journal of Mathematical Analysis and Applications, vol. 332, pp. 258-266, 2012.

[8] S. Sedghi, N. Shobe, and A. Aliouche, "A generalization of fixed point theorems in S-metric spaces," Matematicki Vesnik, vol. 64, pp. 258-266, 2012.

[9] I. A. Bakhtin, "The contraction mapping principle in almost metric spaces," Functional Analysis, vol. 30, pp. 26-37, 1989.

[10] S. Czerwik, "Nonlinear set-valued contraction mappings inbmetric spaces," Atti del Seminario Matematico e Fisico dell'Universita di Modena e Reggio Emilia, vol. 46, no. 2, pp. 263-276, 1998.

[11] V. Parvaneh and S. J. Hosseini Ghoncheh, "Fixed points of $(\psi, \varphi)$-contractive mappings in ordered metric spaces," Global Analysis and Discrete Mathematics, vol. 4, no. 1, pp. 15-29, 2019.

[12] Y. I. Alber and S. Guerre-Delabriere, "Principle of weakly contractive maps in Hilbert spaces," in New Results in Operator Theory and Its Applications, I. Gohberg and Yu. Lyubich, Eds., Birkhauser Verlag, Basel, Switzerland, pp. 7-22, 1997.

[13] B. E. Rhoades, "Some theorems on weakly contractive maps," Nonlinear Analysis: Theory, Methods \& Applications, vol. 47, no. 4, pp. 2683-2693, 2001.

[14] Q. Zhang and Y. Song, "Fixed point theory for generalized $\varphi$-weak contractions," Applied Mathematics Letters, vol. 22, pp. 75-78, 2009.

[15] M. Eshaghi Gordji, M. Ramezani, M. D. L. Sen, and Y. J. Cho, "On orthogonal sets and Banach's fixed point theorem," Fixed Point Theory, vol. 18, no. 2, pp. 569-578, 2017.

[16] B. Samet, C. Vetro, and P. Vetro, "Fixed point theorems for -contractive type mappings," Nonlinear Analysis: Theory, Methods \& Applications, vol. 75, no. 4, pp. 2154-2165, 2012.

[17] A. Gholidahneh and S. Sedghi, "Tripled coincidence point results for $(\psi, \varphi)$-weakly contractive mappings in partially ordered $S_{b}$-metric spaces," Journal of Mathematices Research, vol. 9 , no. $5,2017$.

[18] Z. Mustafa, R. J. Shahkoohi, V. Parvaneh, Z. Kadelburg, and M. M. M. Jaradat, "Modified ordered $S$-metric spaces and some fixed point theorems for contractive mappings with application to periodic boundary value problems," Fixed Point Theory and Applications, vol. 1, pp. 1-20, 2019.

[19] L. Ciric, V. Parvaneh, and N. Hussain, "Fixed point results for weakly $\alpha$-admissible pairs," Filomat, vol. 30 , no. 14 , pp. 3697-3713, 2016.

[20] G. Jungck, "Common fixed points for noncontinuous nonself maps on nonmetric space," Far East Journal of Mathematical Sciences, vol. 4, pp. 199-215, 1996. 
[21] M. S. Khan, M. Swaleh, and S. Sessa, "Fixed point theorems by altering distances between the points," Bulletin of the Australian Mathematical Society, vol. 30, no. 1, pp. 1-9, 1984.

[22] J. Esmaily, S. M. Vaezpour, and B. E. Rhoades, "Coincidence point theorem for generalized weakly contractions in ordered metric spaces," Applied Mathematics and Computation, vol. 219, no. 4, pp. 1536-1548, 2012.

[23] M. Rahman, Integral Equations and Their Applications, WIT press, London, UK, 2007.

[24] J. J. Nieto and R. Rodríguez-López, "Existence and uniqueness of fixed point in partially ordered sets and applications to ordinary differential equations," Acta Mathematica Sinica, English Series, vol. 23, no. 12, pp. 2205-2212, 2007. 